Supporting Information

\title{
Supramolecular Coronation of Platinum(II) Complexes by Macrocycles: Structure, Relativistic DFT Calculations, and Biological Effects
}

Martin Sojka, ${ }^{\dagger, \ddagger}$ Jan Chyba, ${ }^{\dagger, \$}$ Shib Shankar Paul, ${ }^{\dagger, \ddagger}$ Karolina Wawrocka, ${ }^{\dagger, \ddagger}$ Kateřina Hönigová, ${ }^{\$}$

Ben Joseph R. Cuyacot, ${ }^{\dagger \dagger}$ Abril C. Castro, ${ }^{\S}$ Tomáš Vaculovič, ${ }^{\ddagger}$ Jaromír Marek, ${ }^{\dagger}$ Michal Repisky, ${ }^{\Delta}$

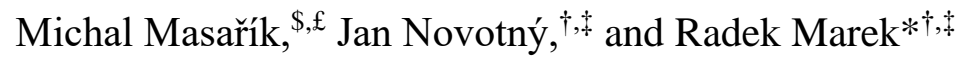

\footnotetext{
${ }^{\dagger}$ CEITEC - Central European Institute of Technology, Masaryk University, Kamenice 5, CZ-62500 Brno, Czechia

$\$$ Department of Chemistry, Faculty of Science, Masaryk University, Kamenice 5, CZ-62500 Brno, Czechia

\$ Department of Pathological Physiology, Faculty of Medicine, Masaryk University, Kamenice 5, CZ-62500 Brno, Czechia

${ }^{\S}$ Hylleraas Centre for Quantum Molecular Sciences, Department of Chemistry, University of Oslo, P.O. Box 1033 Blindern, 0315 Oslo, Norway

${ }^{\Delta}$ Hylleraas Centre for Quantum Molecular Sciences, Department of Chemistry, UiT - The Arctic University of Norway, 9037 Tromsø, Norway

${ }^{£}$ Department of Physiology, Faculty of Medicine, Masaryk University, Kamenice 5, CZ-62500 Brno, Czechia
}

\section{Corresponding Author}

*Email: rmarek@chemi.muni.cz 
Table S1. Experimental ${ }^{195} \mathrm{Pt}$ NMR chemical shifts $\left(\delta^{\exp }\right)$ for compounds $1 \mathbf{a}$ and $2 \mathbf{a}$ in DMF- $d_{7}$ or $\mathrm{D}_{2} \mathrm{O}$ at $298 \mathrm{~K}$. The NMR shifts were referenced relative to $\mathrm{K}_{2} \mathrm{PtCl}_{4}(-1608 \mathrm{ppm})$ in $\mathrm{D}_{2} \mathrm{O}$, and are reported relative to $\mathrm{K}_{2} \mathrm{PtCl}_{6}(0 \mathrm{ppm})$.

\begin{tabular}{lccc}
\hline \multicolumn{1}{c}{ Compound } & \multicolumn{3}{c}{${ }^{\text {195}}$ Pt NMR shift } \\
\hline cisplatin & $\left.-2104^{\mathrm{a}}-d_{7}\right)$ & $\delta^{\exp }\left(\mathrm{D}_{2} \mathrm{O}\right)$ & $\Delta \delta^{\exp }\left(\mathrm{D}_{2} \mathrm{O}-\mathrm{DMF}-d_{7}\right)$ \\
1a & $-2139^{\mathrm{a}}$ & -35 \\
$\mathbf{2 a}$ & -2406 & -2410 & -4 \\
& & -2437 & -7 \\
\hline
\end{tabular}

${ }^{a}$ The ${ }^{195}$ Pt NMR shifts from Reference 1.

Table S2. Calculated (Calc) and experimental (Exp) masses (m/z) of molecular ions in the ESI-MS spectra of compounds $\mathbf{1 a - 7 d}$.

\begin{tabular}{cccc}
\hline Compound & Molecular ion & \multicolumn{2}{c}{ Mass $(\mathrm{m} / \mathrm{z})$} \\
\hline $\mathbf{1 a}$ & {$\left[1 \mathrm{a}-\left(\mathrm{NO}_{3}\right)^{-}\right]^{+}$} & 416.1214 & 416.1220 \\
$\mathbf{2 a}$ & {$\left[2 \mathrm{a}-\left(\mathrm{NO}_{3}\right)^{-}\right]^{+}$} & 416.1214 & 416.1212 \\
$\mathbf{3 a}$ & {$\left[3 \mathrm{a}-(\mathrm{DMF}) \mathrm{H}^{+}\right]^{-}$} & 452.0056 & 452.0052 \\
$\mathbf{4 a}$ & {$[4 \mathrm{a}+\mathrm{Cl}]^{-}$} & 603.1421 & 603.1417 \\
$\mathbf{5 a}$ & {$[5 \mathrm{a}+\mathrm{Cl}]^{-}$} & 603.1421 & 603.1425 \\
$\mathbf{5 b}$ & {$[5 \mathrm{~b}+\mathrm{Cl}]^{-}$} & 659.2011 & 659.2047 \\
$\mathbf{5 c}$ & {$[5 \mathrm{c}+\mathrm{Cl}]^{-}$} & 607.1734 & 607.1735 \\
$\mathbf{7 d}$ & {$\left[7 \mathrm{~d}-(\mathrm{ABCO}) \mathrm{H}^{+}\right]^{-}$} & 411.9742 & 411.9744 \\
\hline
\end{tabular}


$\mathbf{1 a}$ - positive mode, $\mathrm{MeOH}$
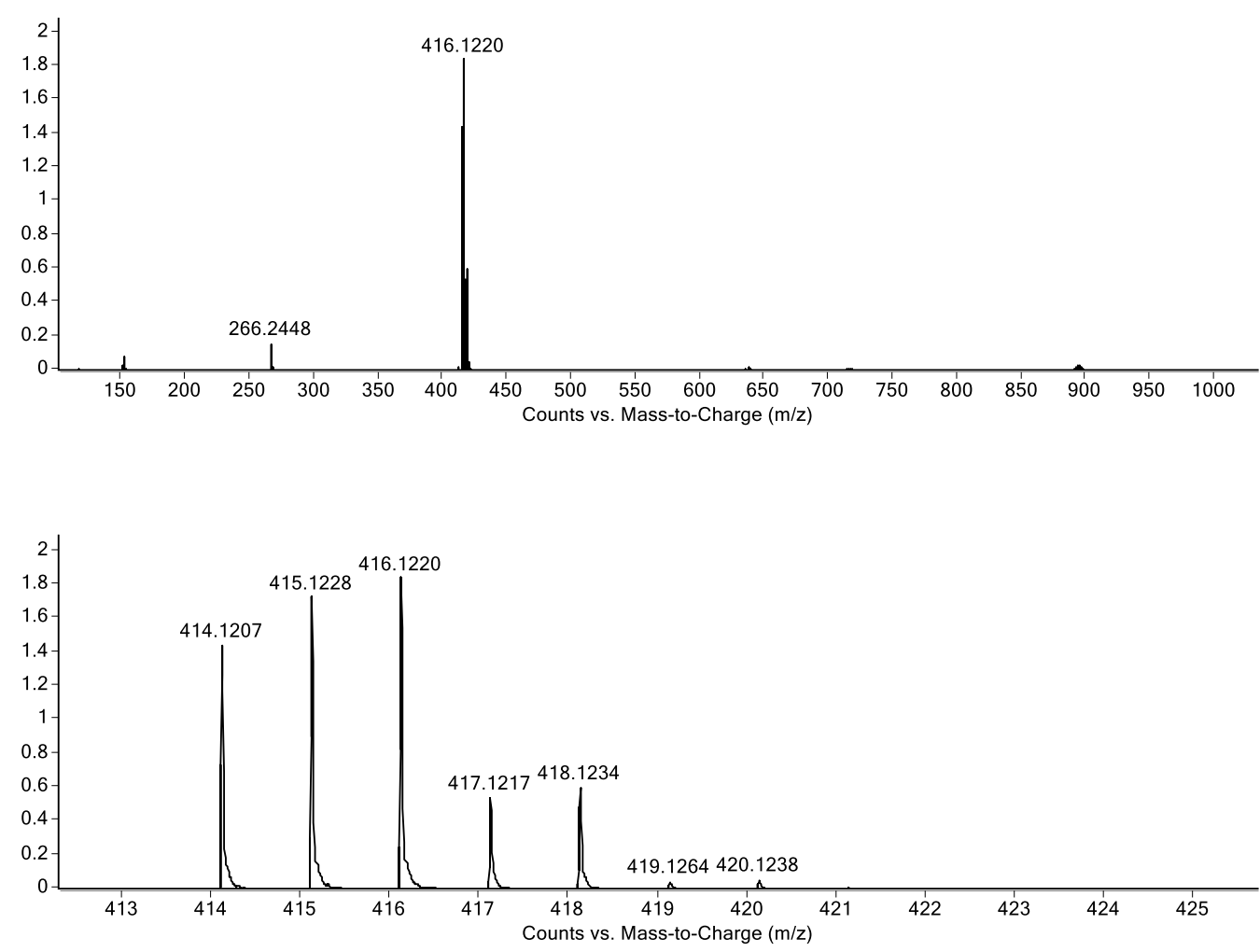

1a_aq@CB7 - positive mode, water
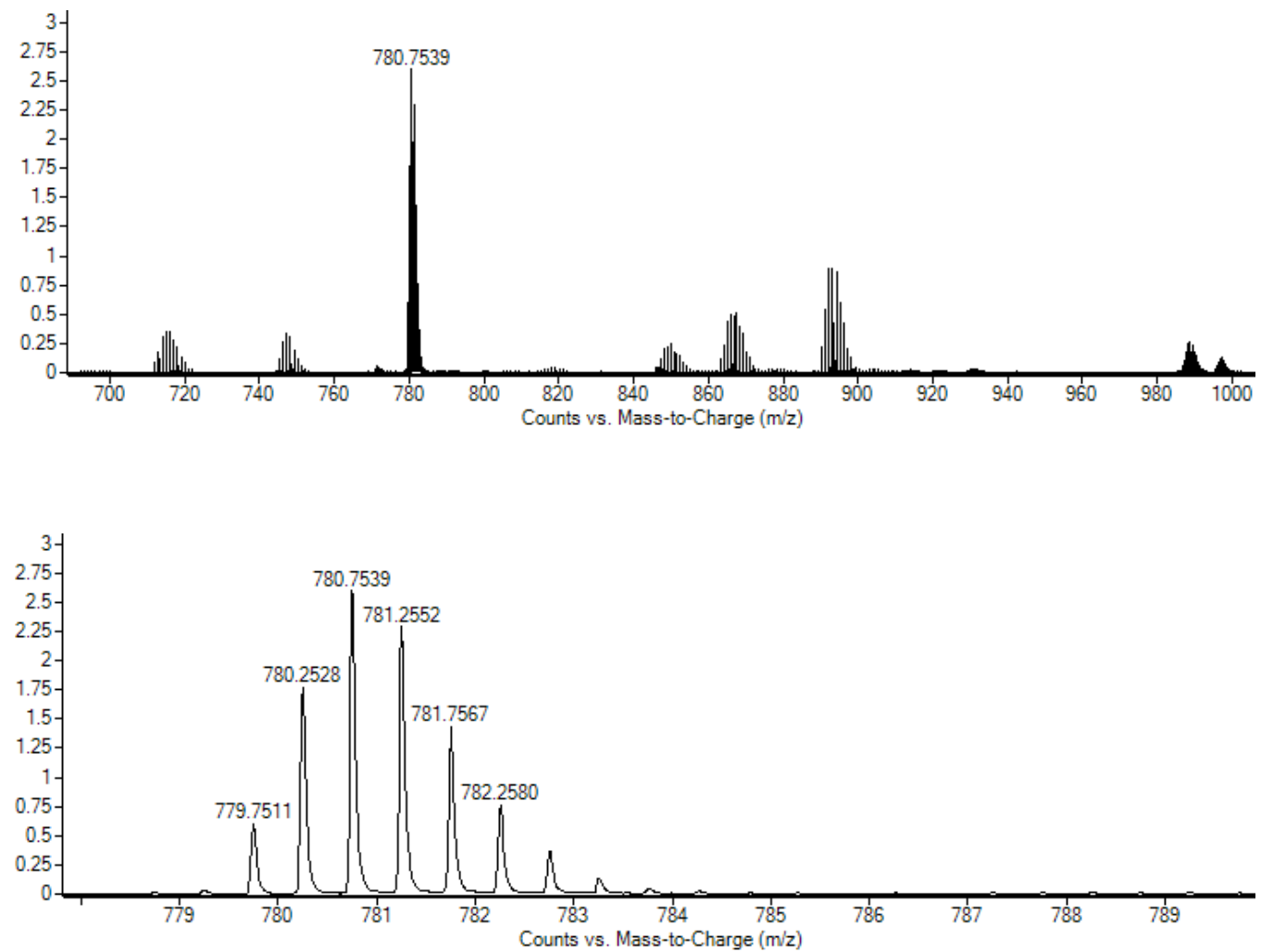

Figure S1a. Results of ESI-MS analyses of compounds 1a and 1a_aq@CB7. 
2a-positive mode, $\mathrm{MeOH}$
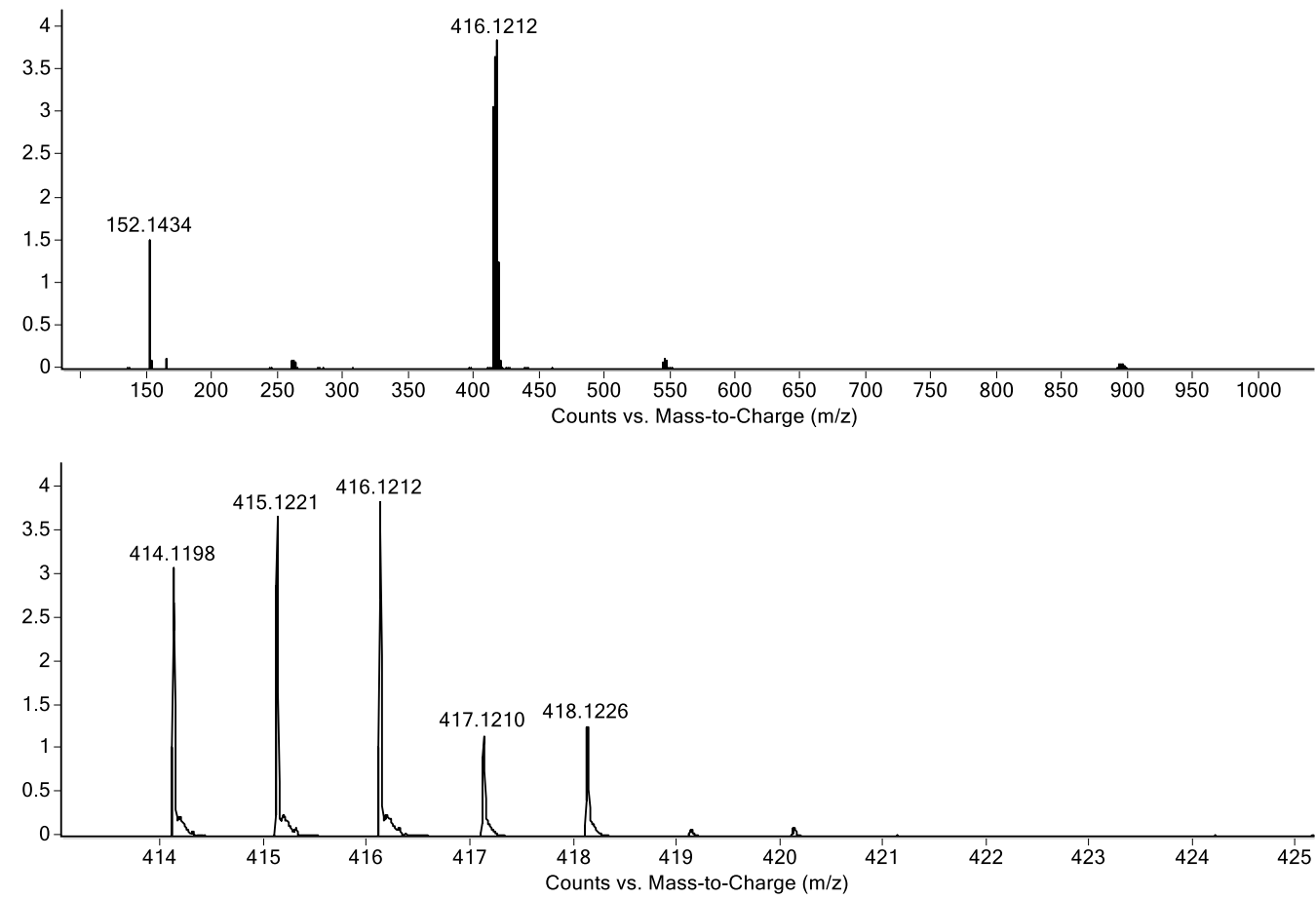

$\mathbf{3 a}$ - negative mode, $\mathrm{MeOH}$
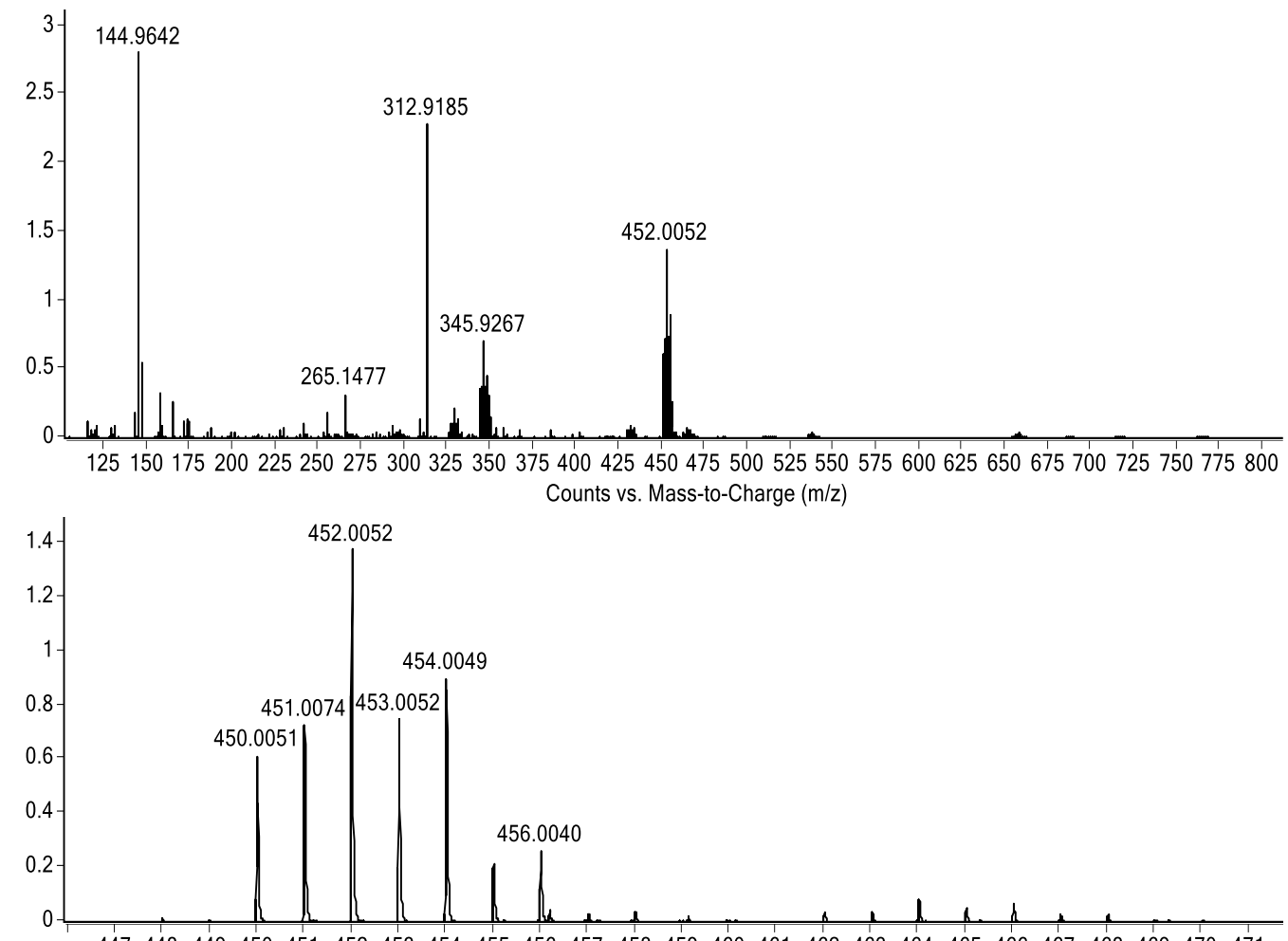

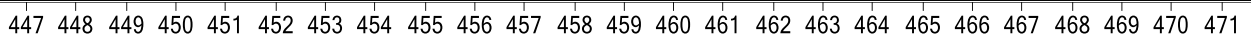
Counts vs. Mass-to-Charge $(\mathrm{m} / \mathrm{z})$

Figure S1b. Results of ESI-MS analyses of compounds 2a and 3a. 
4a - negative mode, $\mathrm{MeOH}$
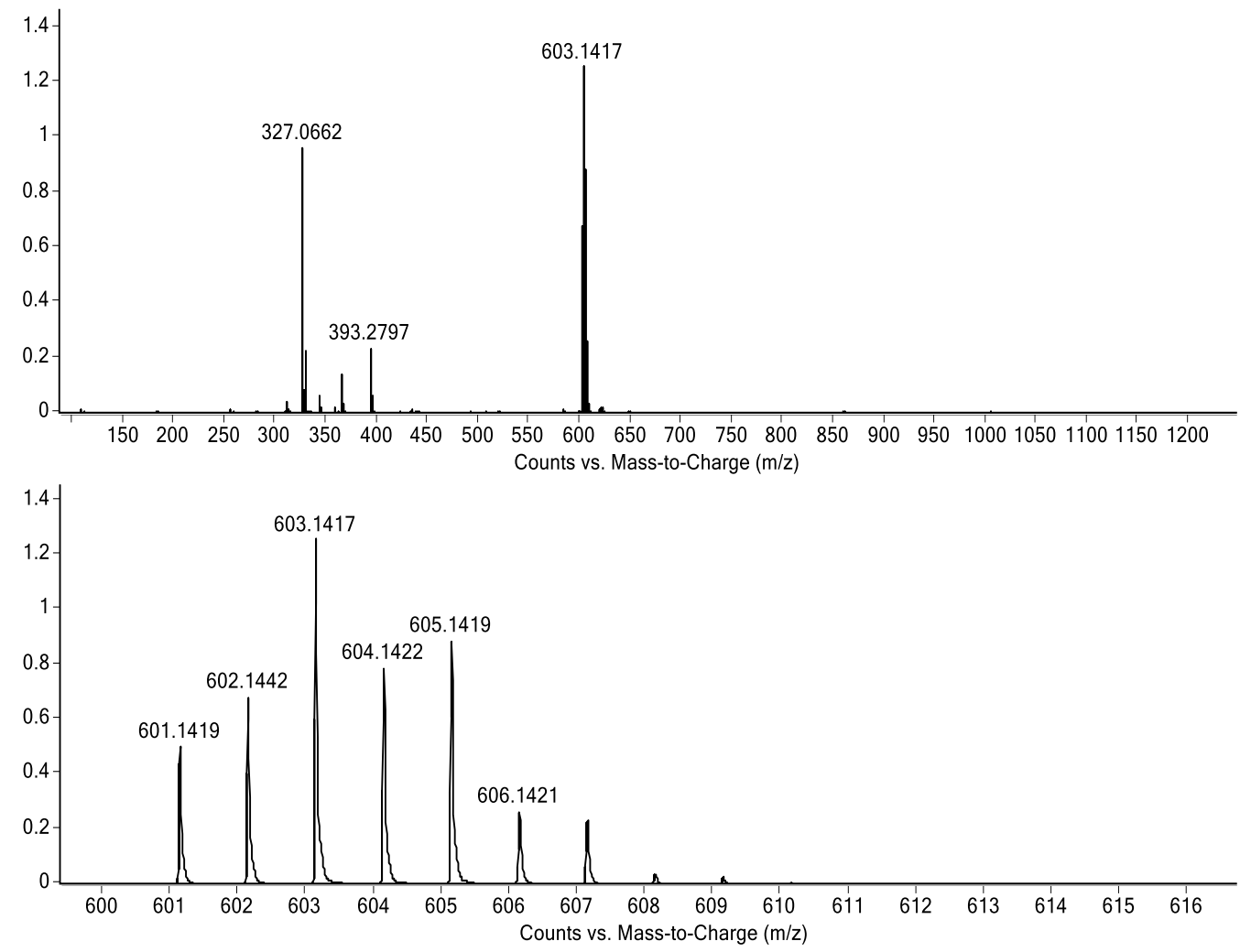

$\mathbf{5 a}$ - negative mode, DMF
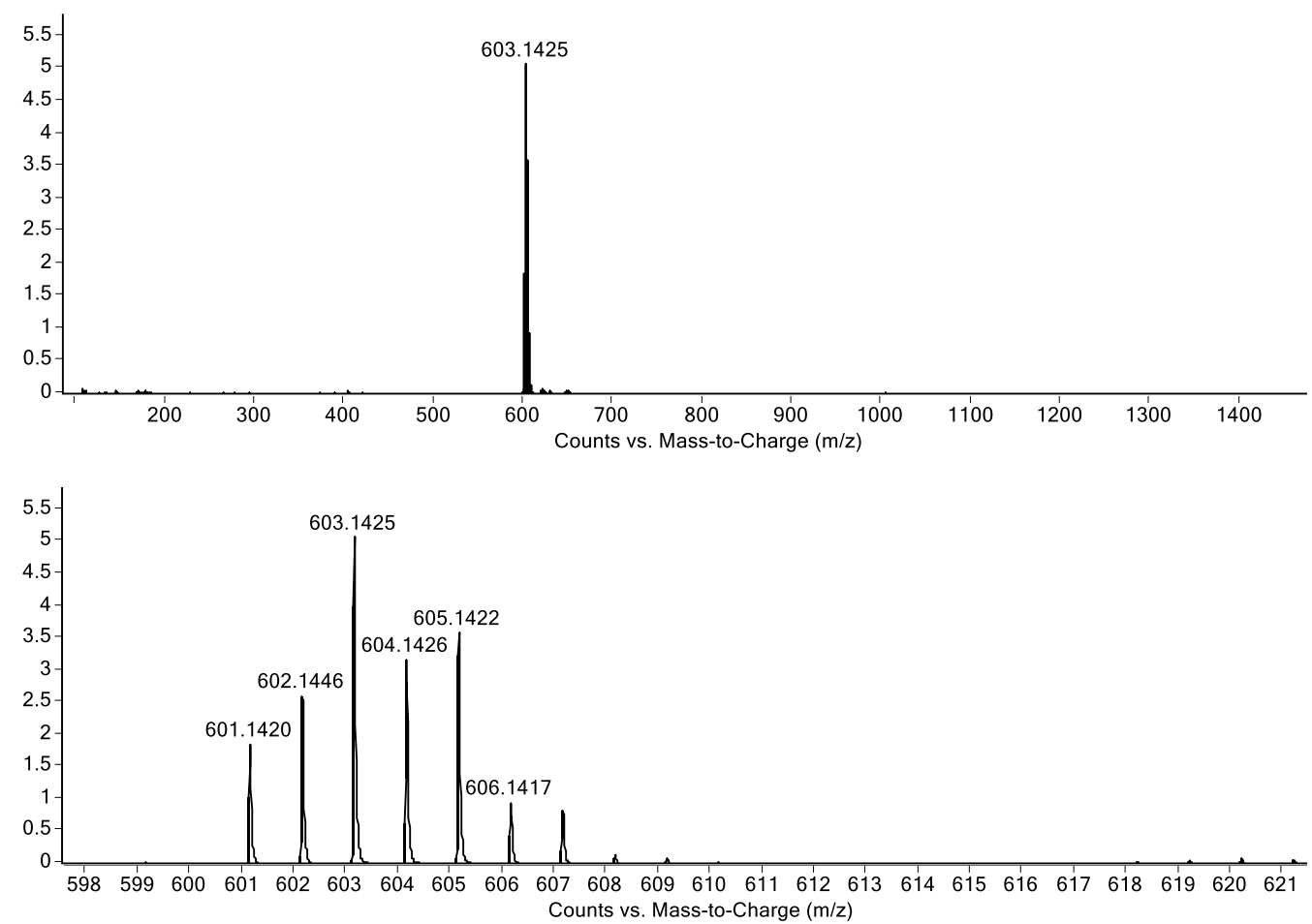

Figure S1c. Results of ESI-MS analyses of compounds 4a and 5a. 


\section{$5 b$ - negative mode, $\mathrm{MeOH}$}
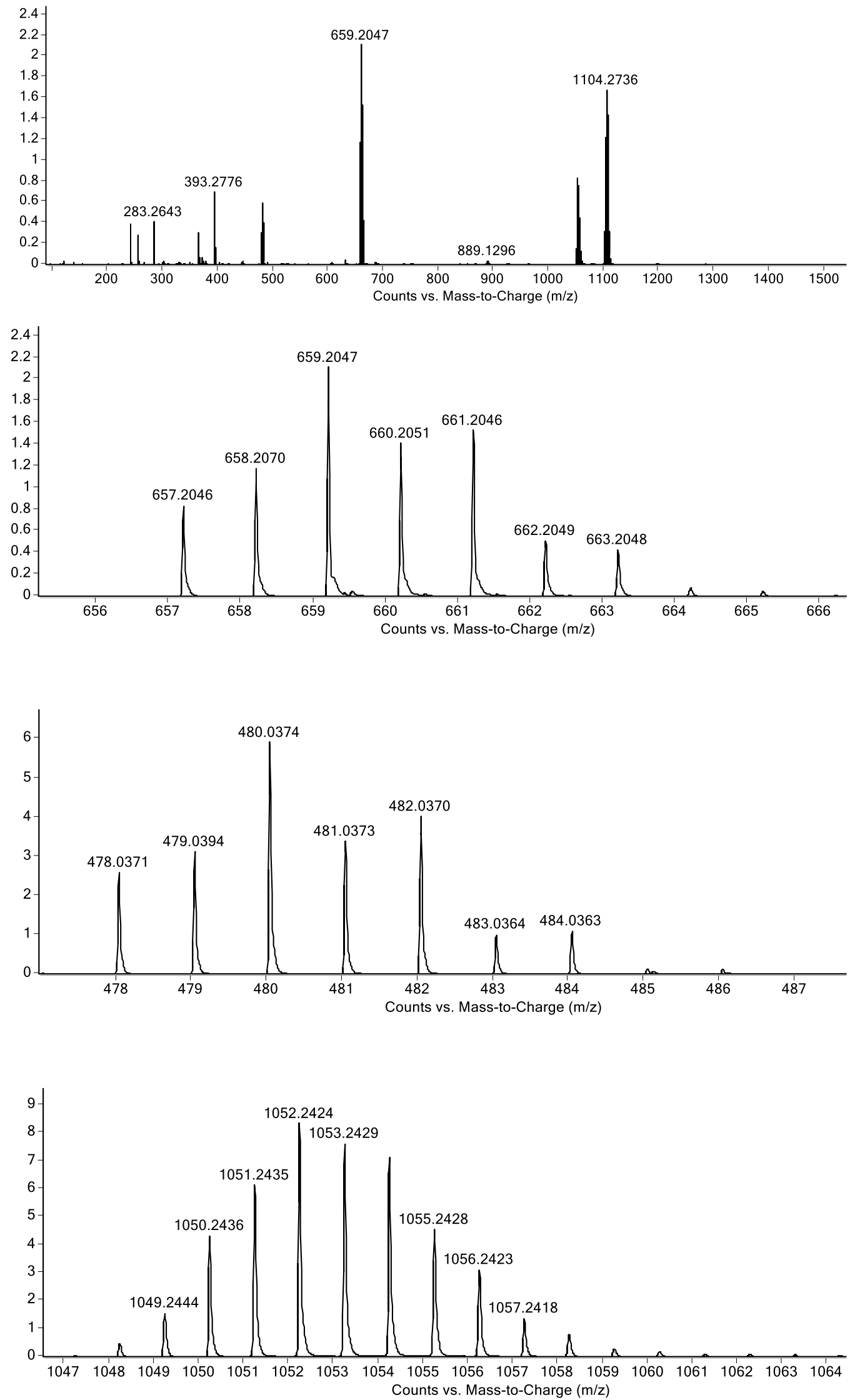

Figure S1d. Results of ESI-MS analyses of compound $\mathbf{5 b}$. 
5c - negative mode, $\mathrm{MeOH}$
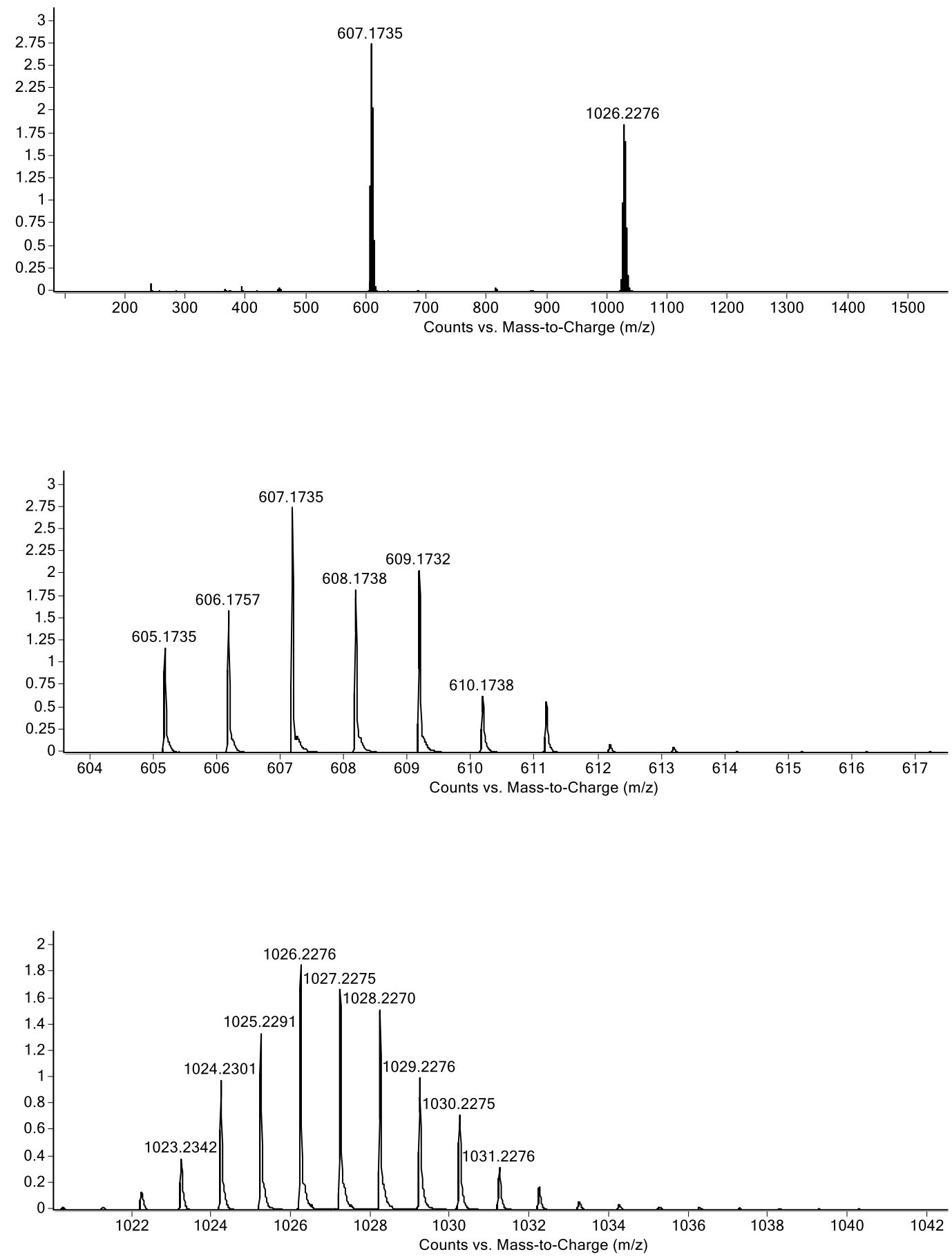

Figure S1e. Results of ESI-MS analyses of compound 5c. 
$\mathbf{6 b}$ - negative mode, $\mathrm{MeOH}$

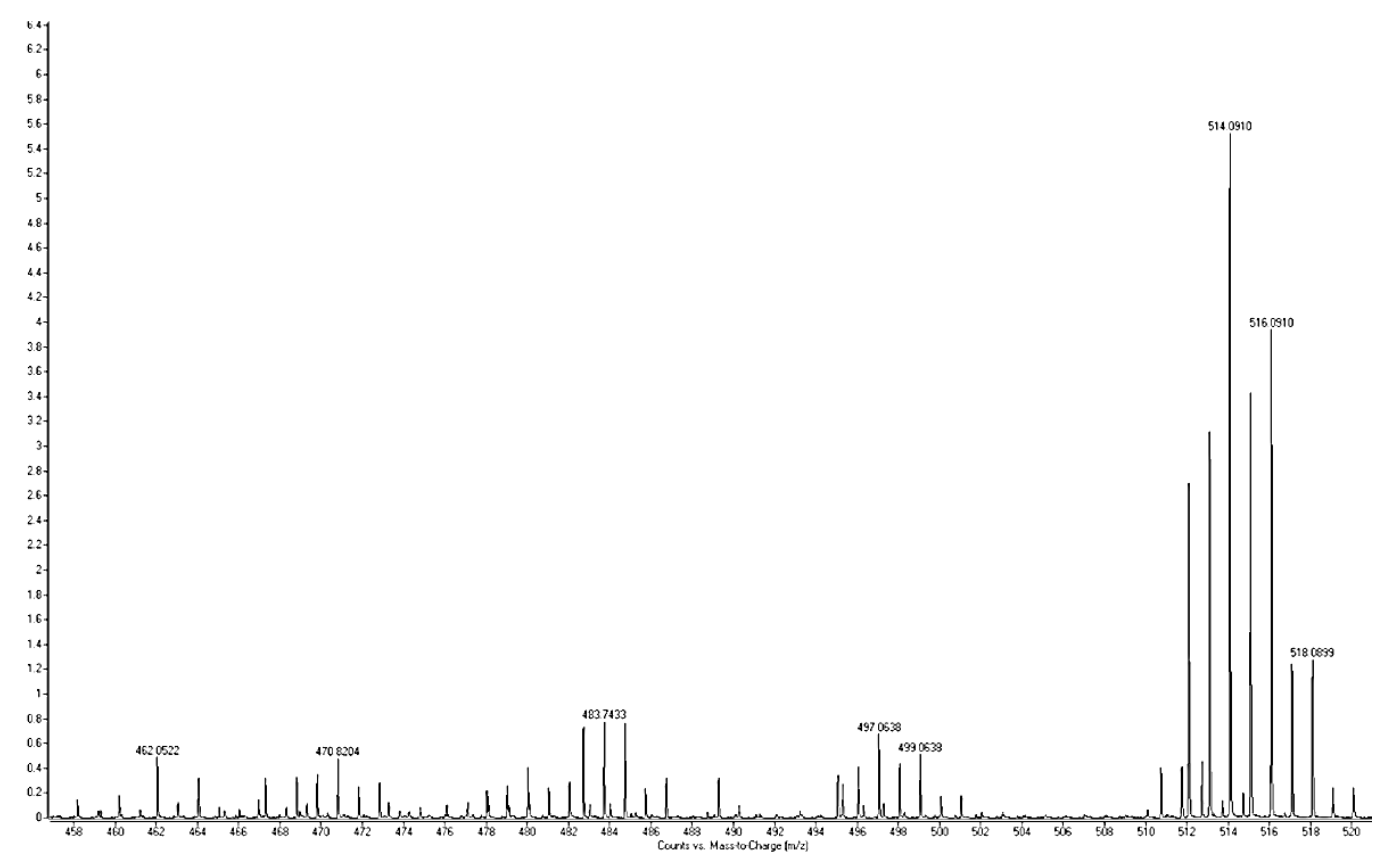

6c - positive mode, $\mathrm{MeOH}$
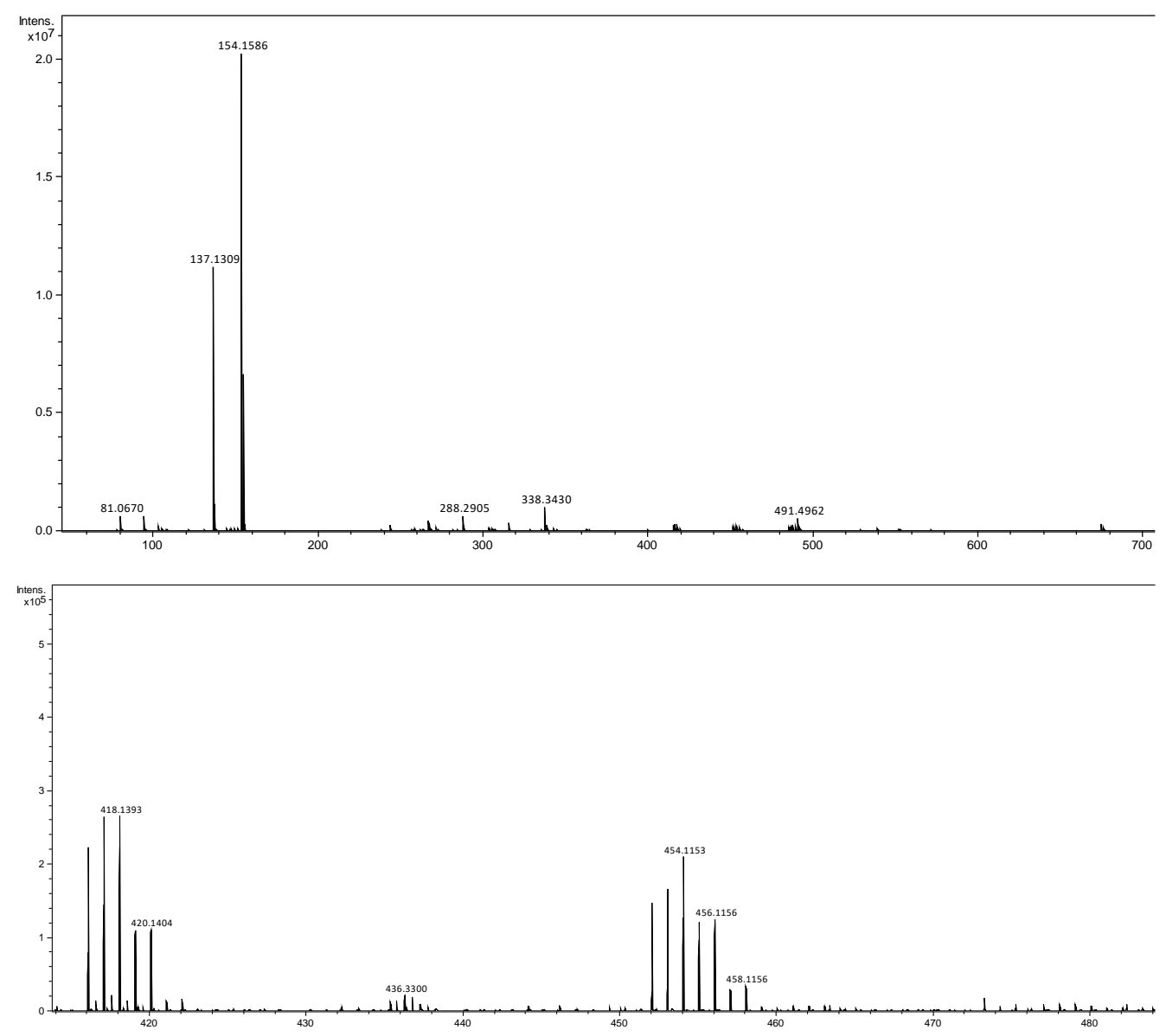

Figure S1f. Results of ESI-MS analyses of compounds $\mathbf{6 b}$ and $\mathbf{6 c}$. 


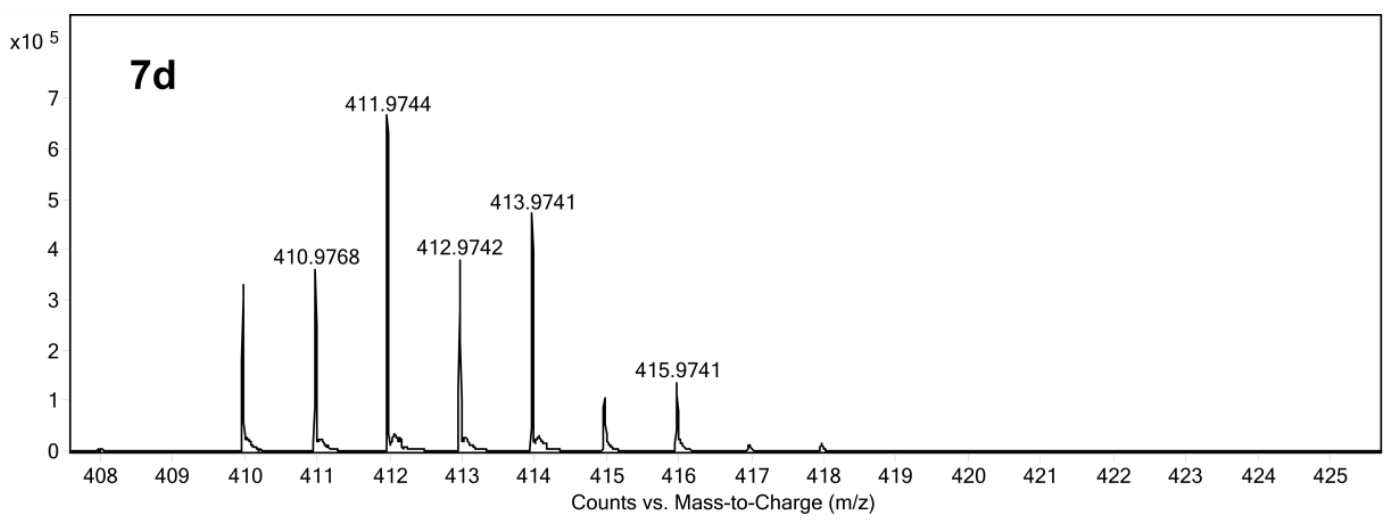

Figure S1g. Results of ESI-MS analyses of the compound 7d. 
(a)

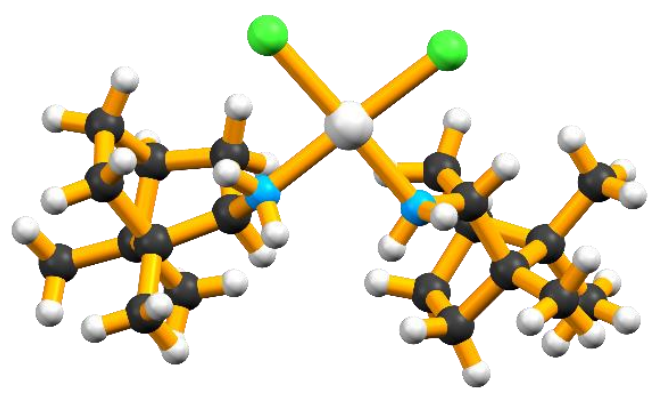

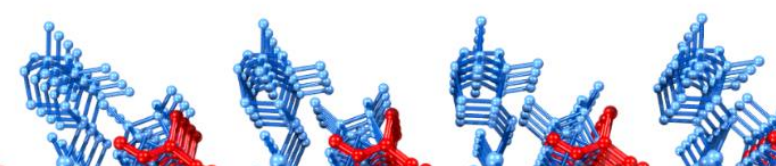

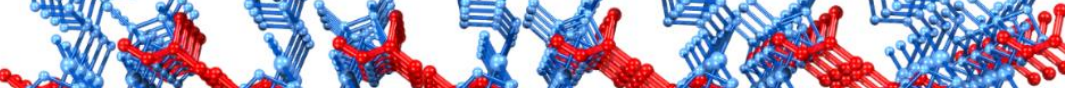

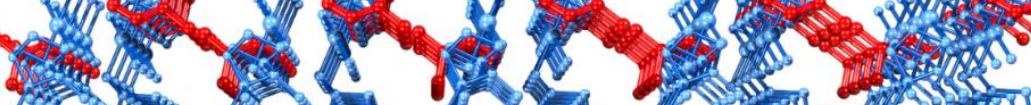

livin

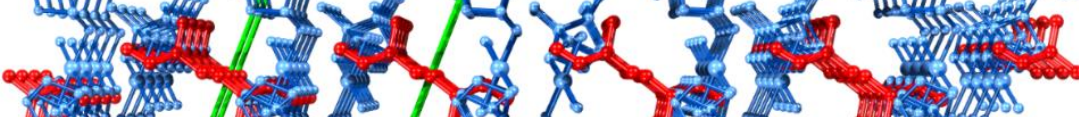

sa.

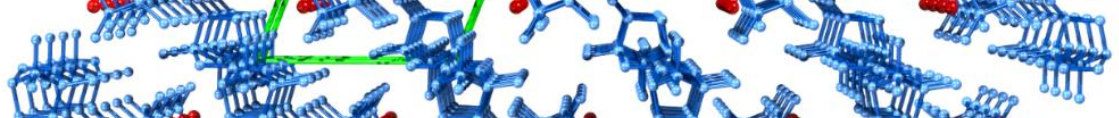

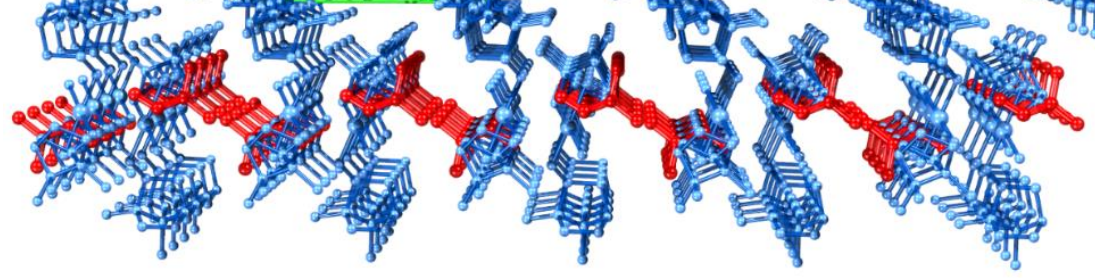

(b)

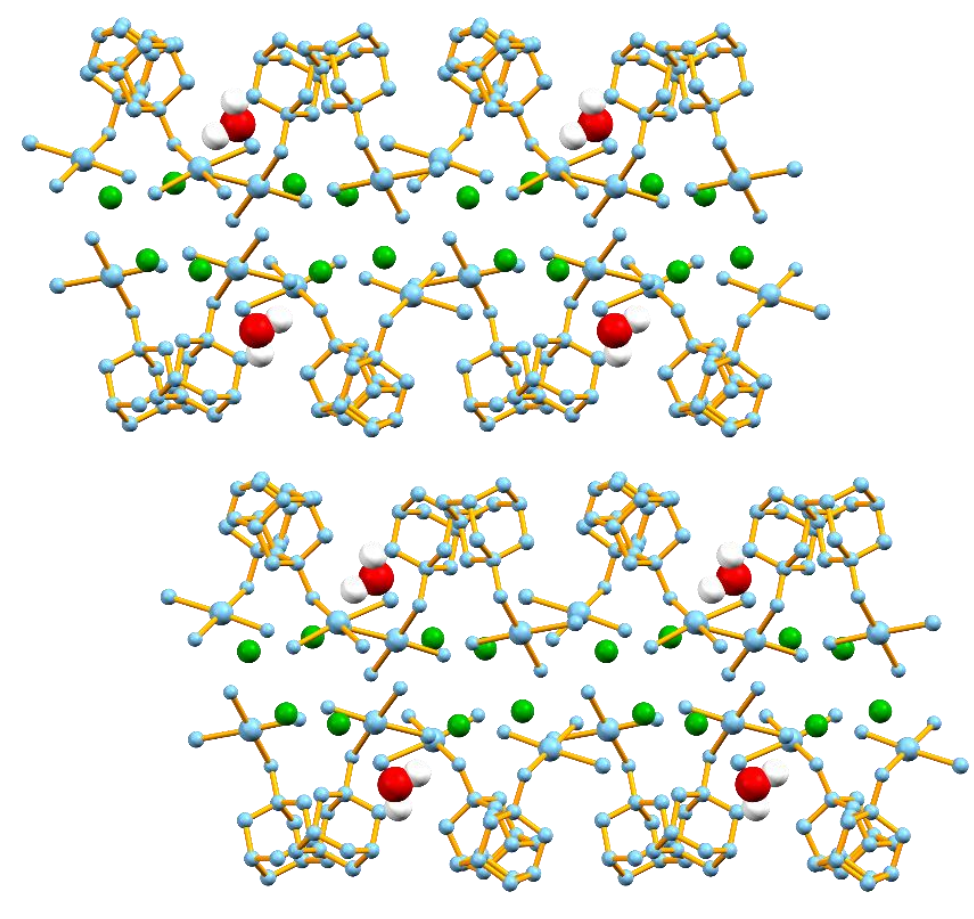



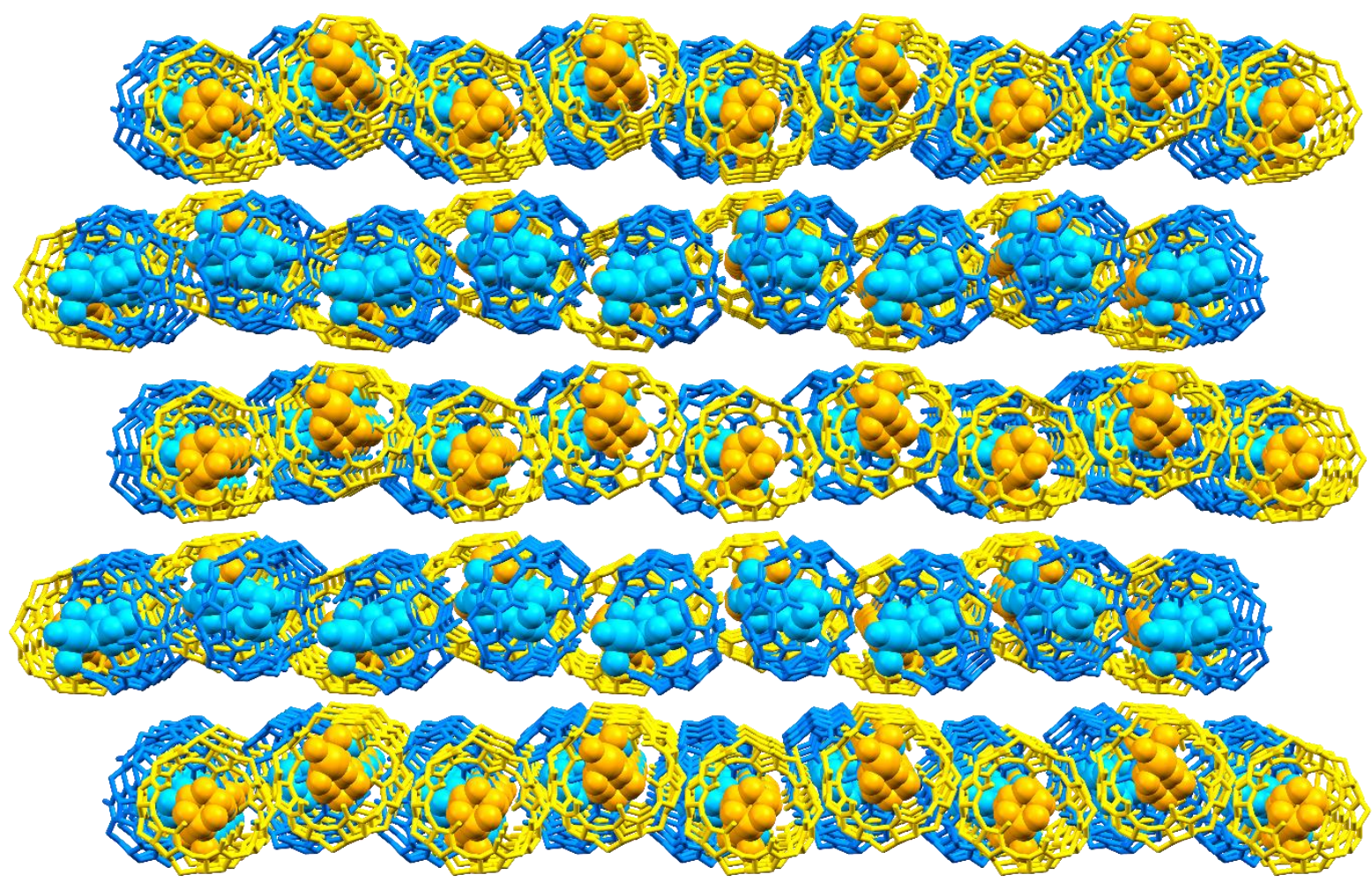

(c)

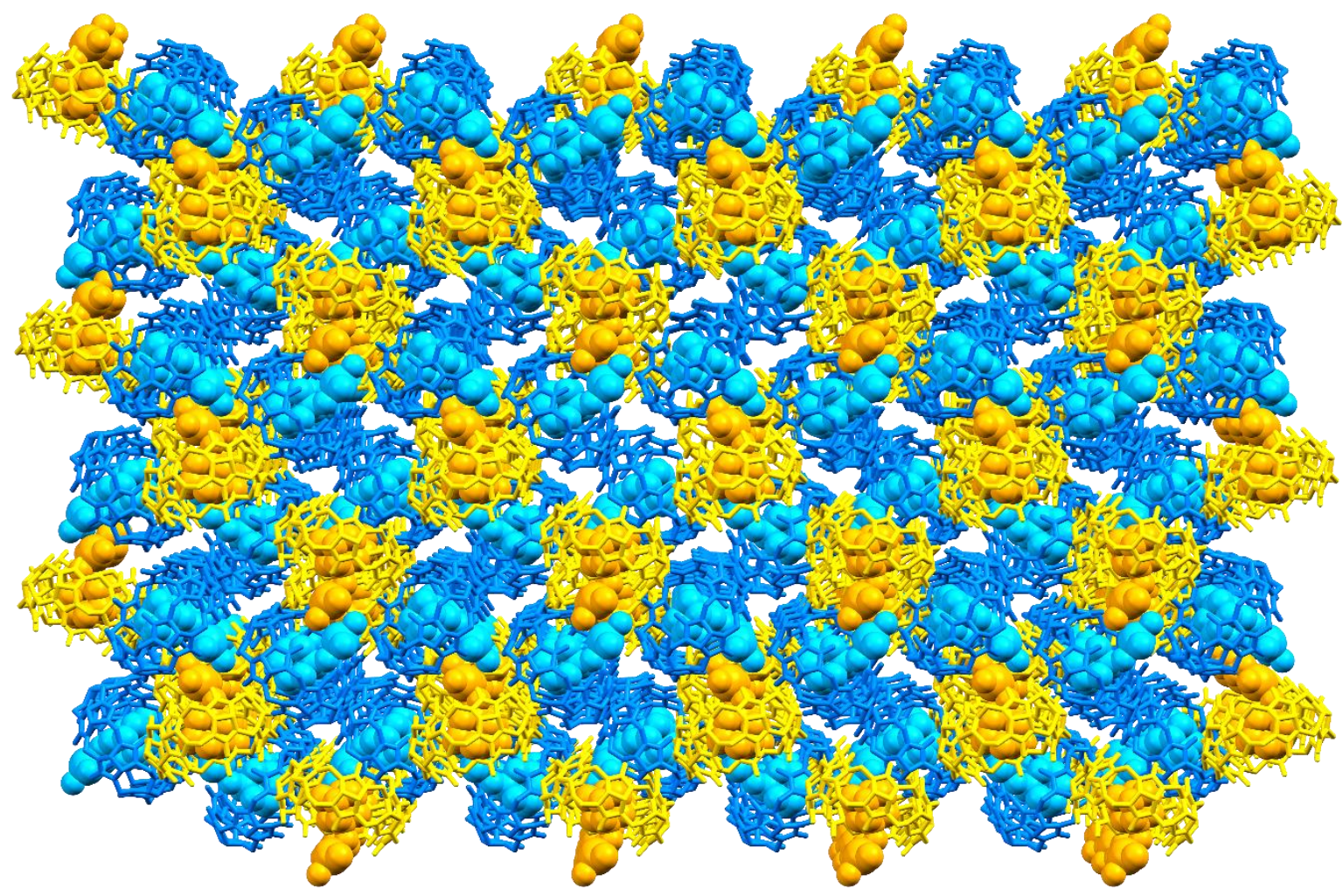


(d)

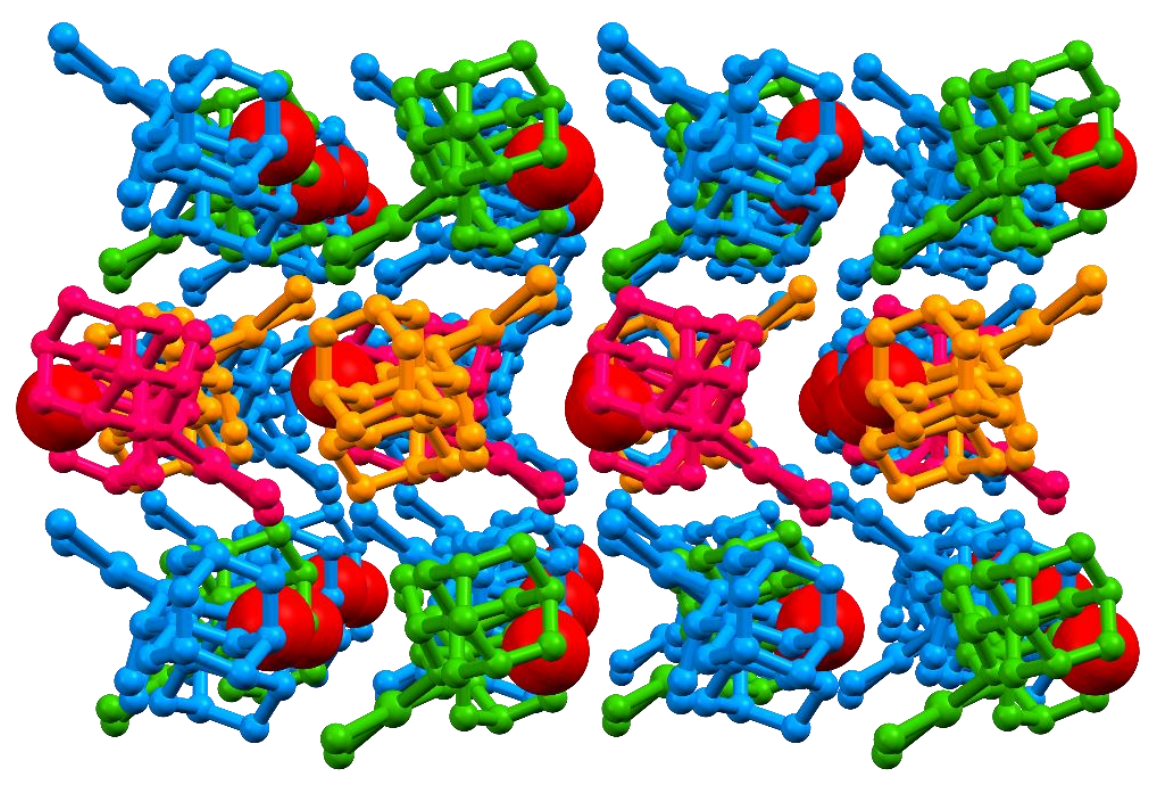

(e)

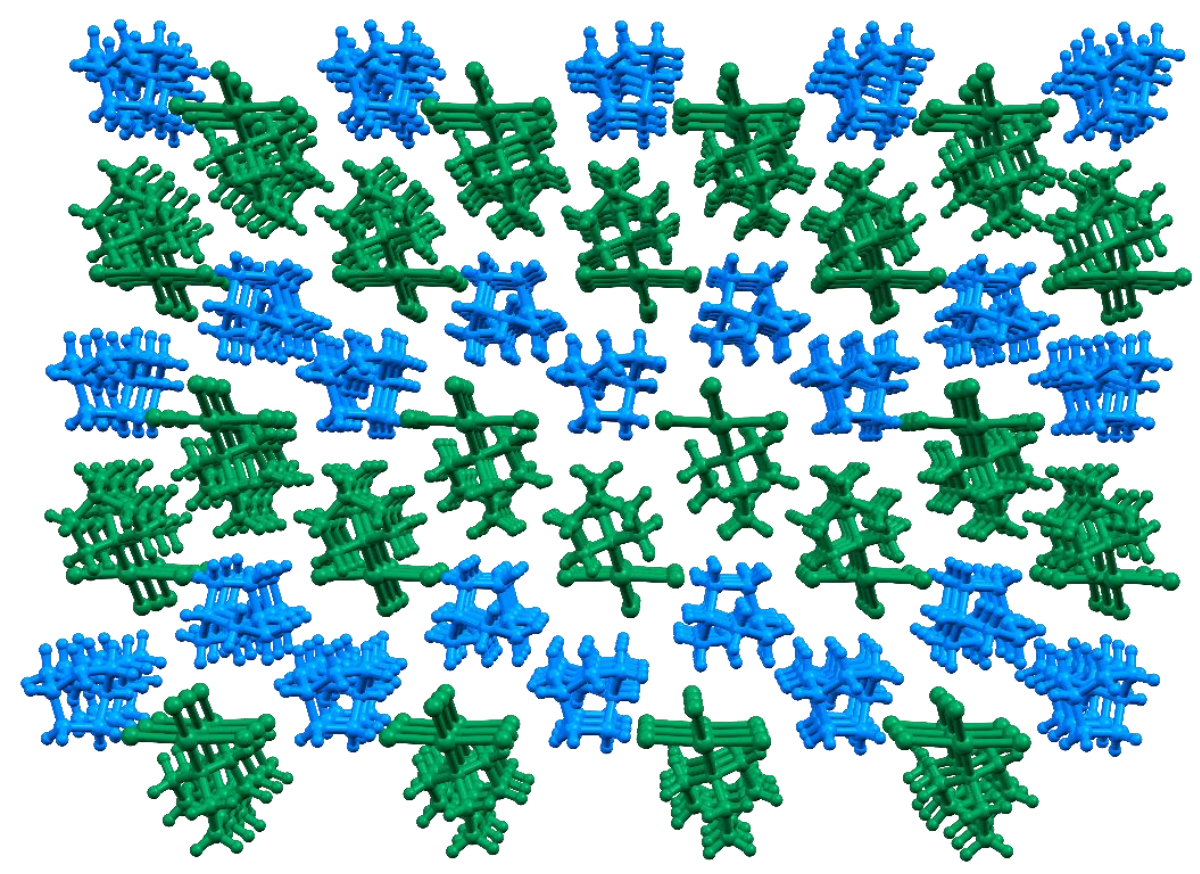

Figure S2. (a) Molecular structure of compound 5c. Crystal packing of (b) 1a, (c) 1a@CB7, (d) 5b, and (e) 7d. 
Table S3. Selected interatomic distances and bond angles of the compounds analyzed by X-ray diffraction.

\begin{tabular}{lccccccccc}
\hline Compound & Pt-N2 & Pt-X & Pt-Y & Pt-Z & N2-Pt-X & X-Pt-Y & Y-Pt-Z & N2-Pt-Z & mdev \\
\hline $\mathbf{1 a}$ & $2.049(6)$ & $2.310(2)$ & $2.044(7)$ & $2.044(5)$ & 89.41 & 90.37 & 88.83 & 91.34 & 0.028 \\
$\mathbf{1 a} @ \mathbf{C B 7}$ & $2.084(5)$ & $2.131(6)$ & $2.021(6)$ & $2.012(6)$ & 93.89 & 84.16 & 90.71 & 91.36 & 0.019 \\
$\mathbf{5 b}$ & $2.027(8)$ & $2.305(3)$ & $2.309(3)$ & $2.057(8)$ & 89.29 & 89.73 & 88.80 & 92.12 & 0.037 \\
$\mathbf{5 c}$ & $2.055(4)$ & $2.317(1)$ & $2.319(1)$ & $2.059(3)$ & 86.97 & 93.78 & 87.82 & 91.41 & 0.020 \\
$\mathbf{7 d}$ & $2.109(2)$ & $2.3109(7)$ & $2.3159(7)$ & $2.3093(7)$ & 90.17 & 88.54 & 87.10 & 94.37 & 0.011 \\
\hline
\end{tabular}

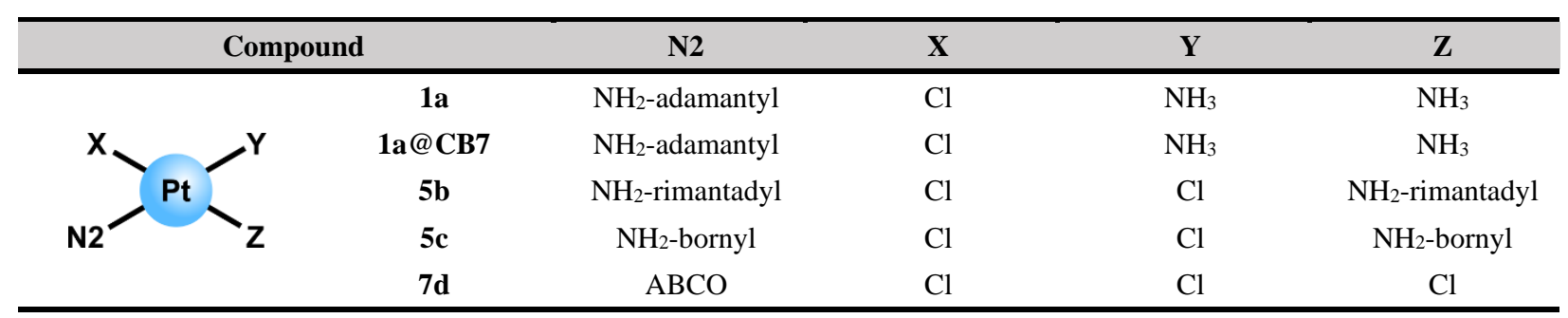



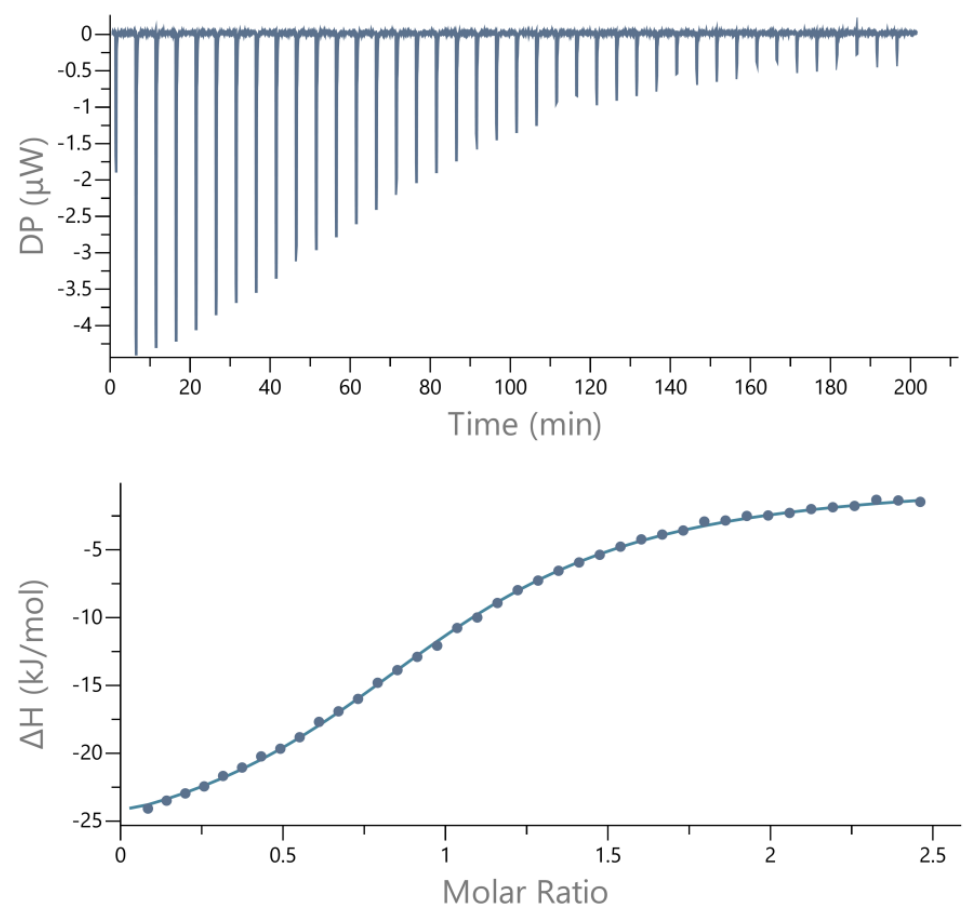

Figure S3a. The ITC direct experiment of the interaction between compound 2a and $\beta$-CD in Milli- $\mathrm{Q}^{\circledR}$ water at $298 \mathrm{~K}$ showing the differential power (DP) over time (top) and enthalpy change $(\Delta \mathrm{H})$ over HG stoichiometry (bottom).
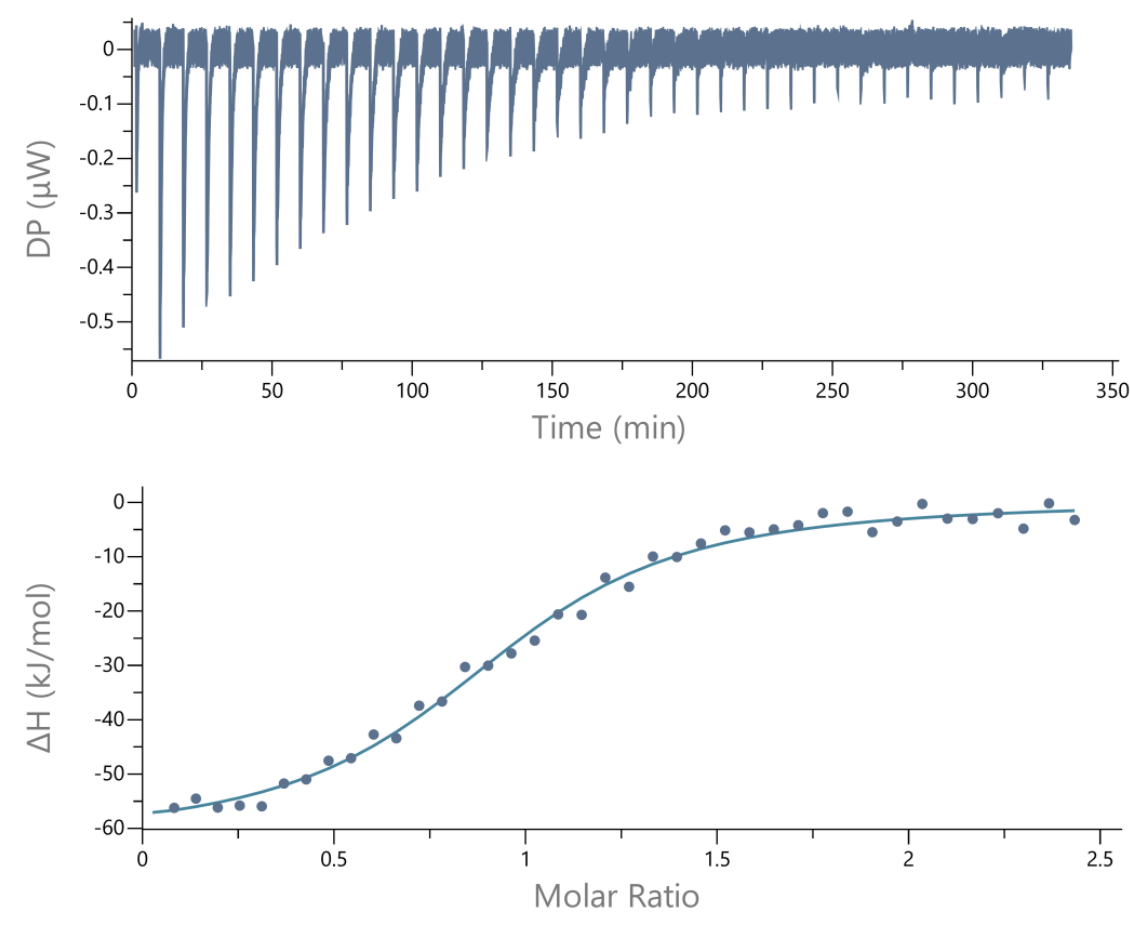

Figure S3b. The ITC competitive experiment of the interaction between compound 2a and CB7 in Milli- ${ }^{\circledR}$ water at $298 \mathrm{~K}$ showing the differential power (DP) over time (top) and enthalpy change $(\Delta \mathrm{H})$ over HG stoichiometry (bottom). 
Table S4. Stoichiometry (n) and thermodynamic parameters (enthalpy, $\Delta H$, entropy, $\mathbf{T} \Delta S$, and Gibbs energy, $\Delta \boldsymbol{G}$ ) of the supramolecular binding between compound 2a and the $\beta$-CD or CB7 macrocyclic host as determined by ITC measurement in Milli- ${ }^{\circledR}$ water at $298 \mathrm{~K}$.

\begin{tabular}{|c|c|c|c|c|}
\hline Host & $\bar{n}$ & $\Delta H\left[\mathrm{~kJ} \mathrm{~mol}^{-1}\right]$ & $\mathrm{T} \Delta S\left[\mathrm{~kJ} \mathrm{~mol}^{-1} \mathrm{~K}^{-1}\right]$ & $\Delta G\left[\mathrm{~kJ} \mathrm{~mol}^{-1}\right]$ \\
\hline$\beta-\mathrm{CD}$ & $1.02 \pm 0.01$ & $-27.9 \pm 0.2$ & -1.2 & -26.7 \\
\hline CB7 & $0.97 \pm 0.02$ & $-94.9 \pm 0.2$ & -32.9 & -62.0 \\
\hline
\end{tabular}




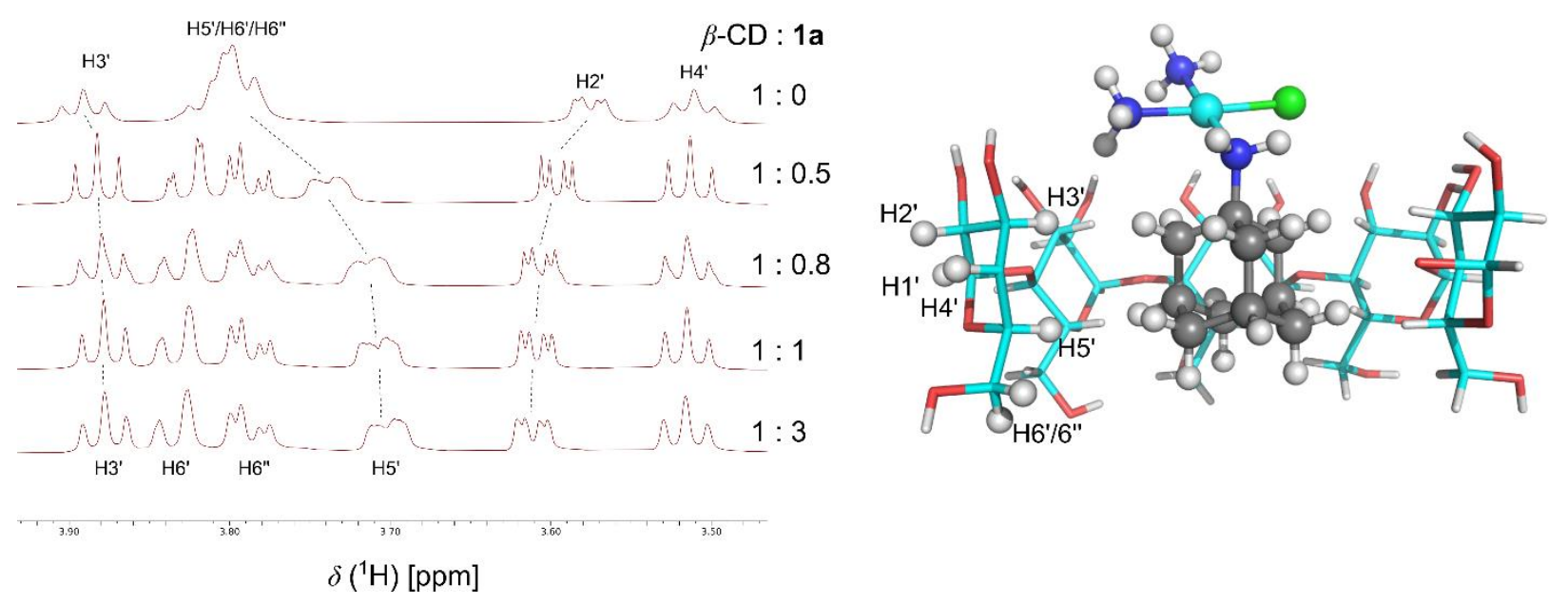

Figure S4a. ${ }^{1} \mathrm{H}$ NMR titration of $1 \mathrm{a}$ into a solution of $\beta$-CD performed in $\mathrm{D}_{2} \mathrm{O}$ at $293 \mathrm{~K}$. The atom numbering scheme for $\beta$-CD is shown on the structural representation of complex $1 \mathbf{a} @ \boldsymbol{\beta}$-CD.

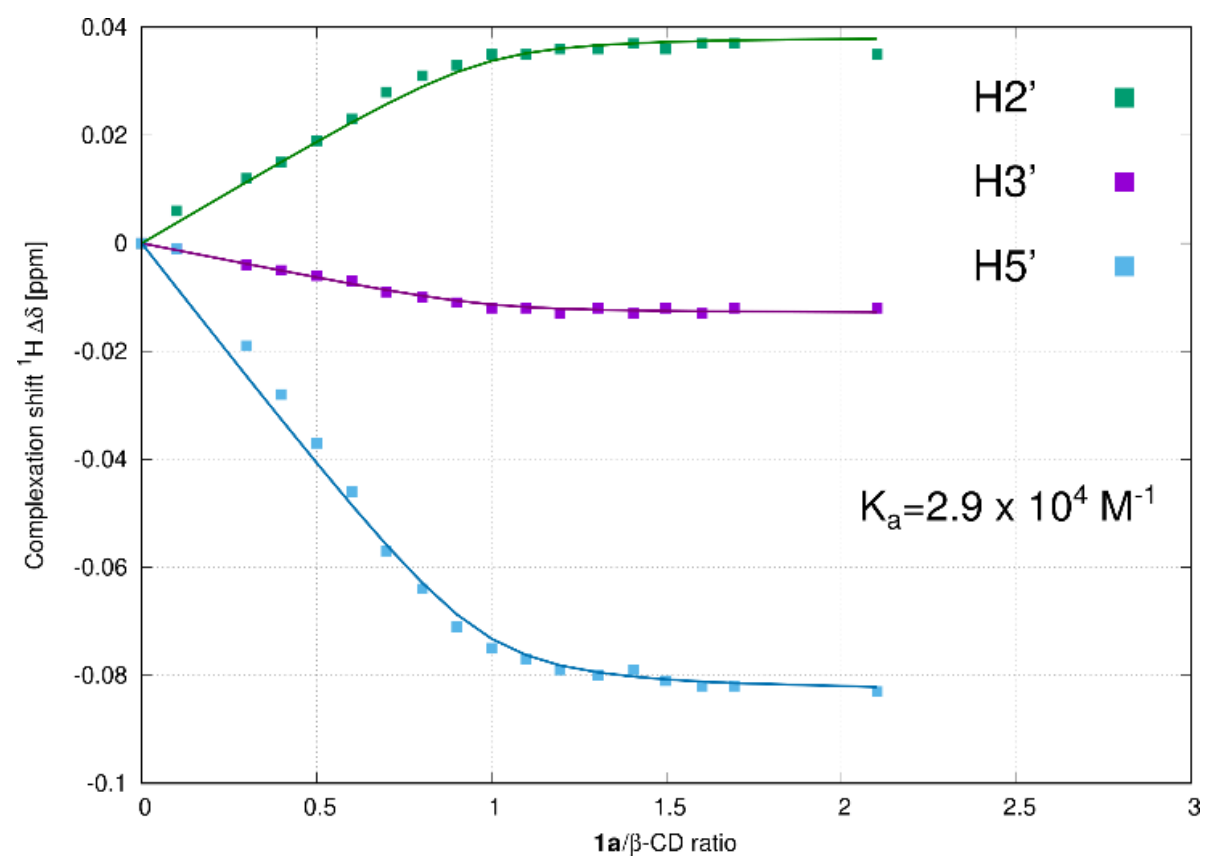

Figure S4b. Complexation-induced ${ }^{1} \mathrm{H}$ NMR shifts of $\beta$-CD in the course of titration with 1a. Experimental points were fitted using a 1:1 model in HypNMR software (solid lines). ${ }^{2}$ 


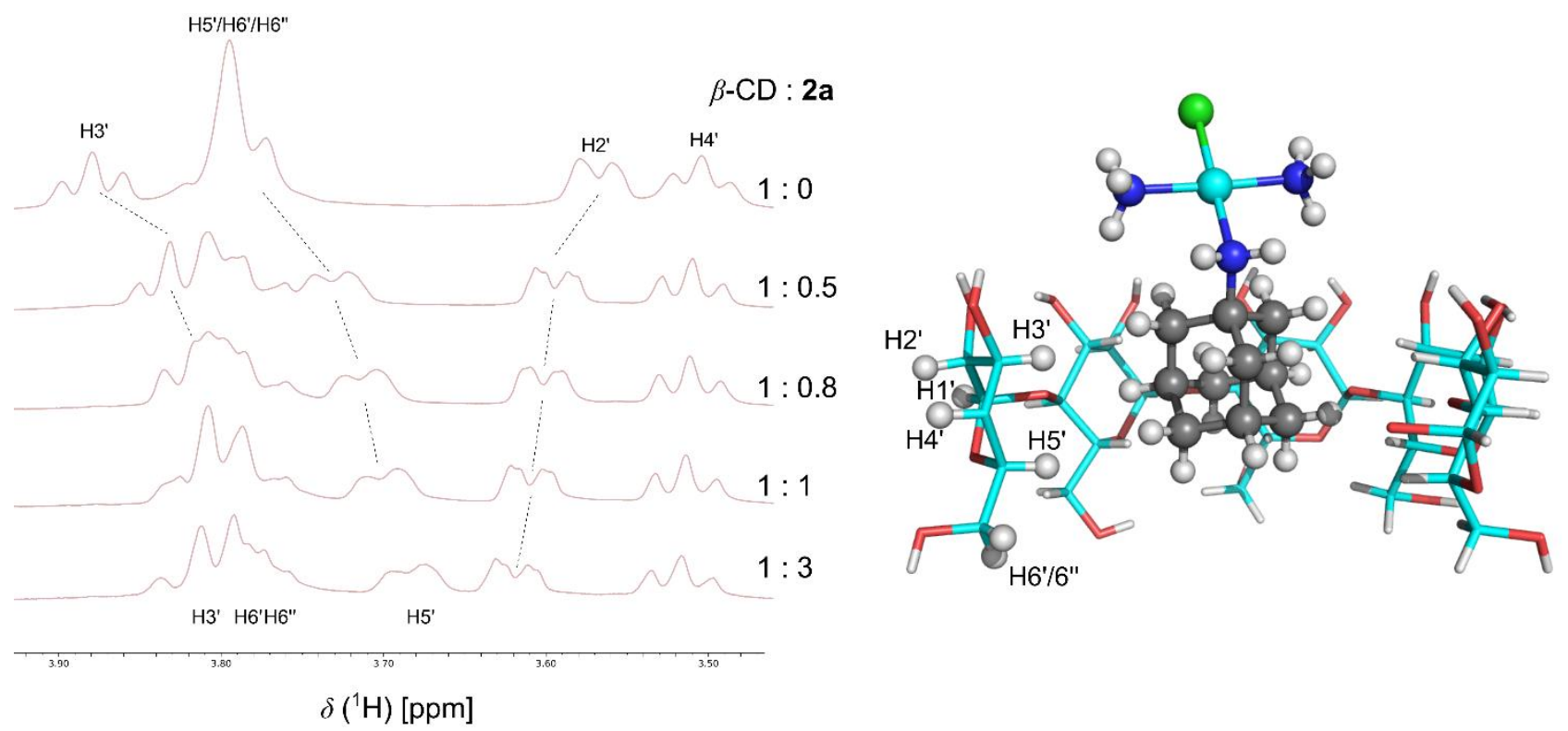

Figure S4c. ${ }^{1} \mathrm{H}$ NMR titration of $2 \mathbf{a}$ into a solution of $\beta$-CD performed in $\mathrm{D}_{2} \mathrm{O}$ at $293 \mathrm{~K}$.

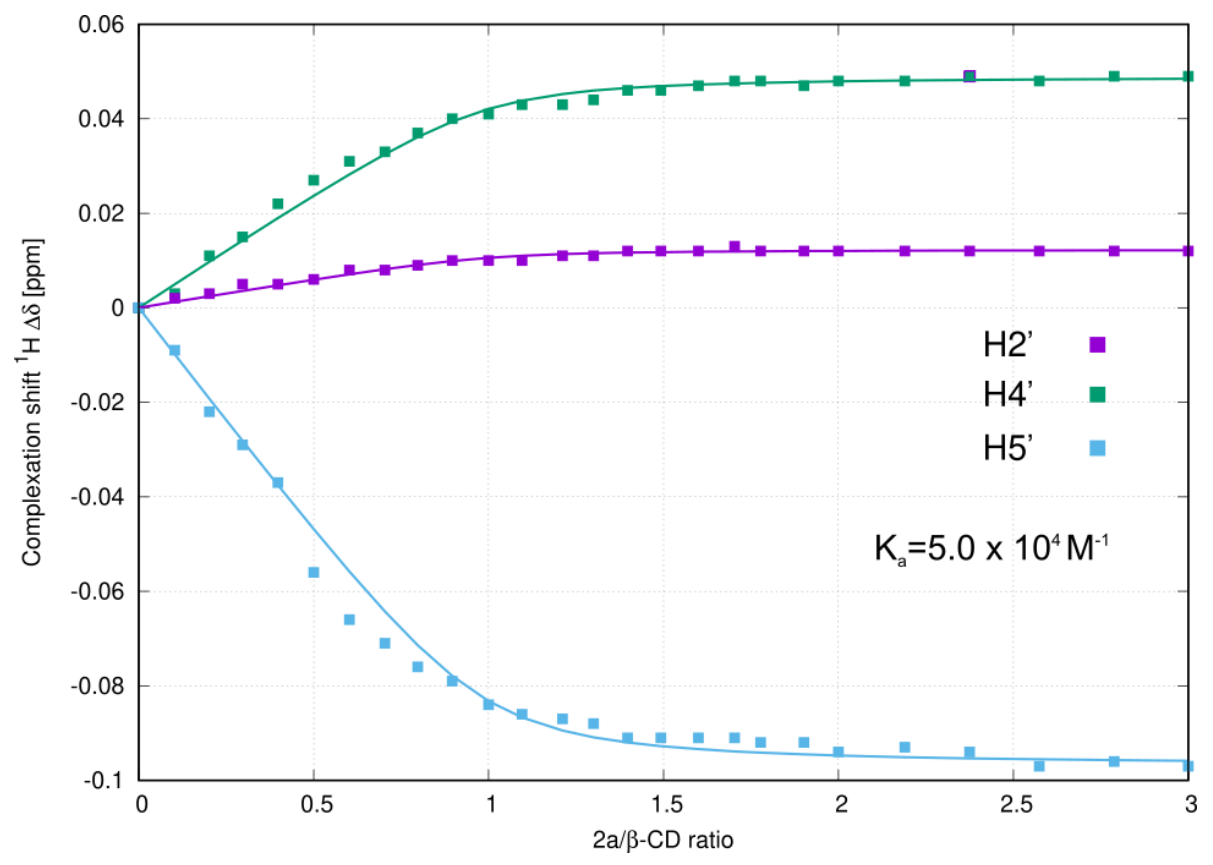

Figure S4d. Complexation-induced ${ }^{1} \mathrm{H}$ NMR shifts of $\beta$-CD in the course of titration with 2a. Experimental points were fitted using a 1:1 model in HypNMR software (solid lines). ${ }^{2}$ 

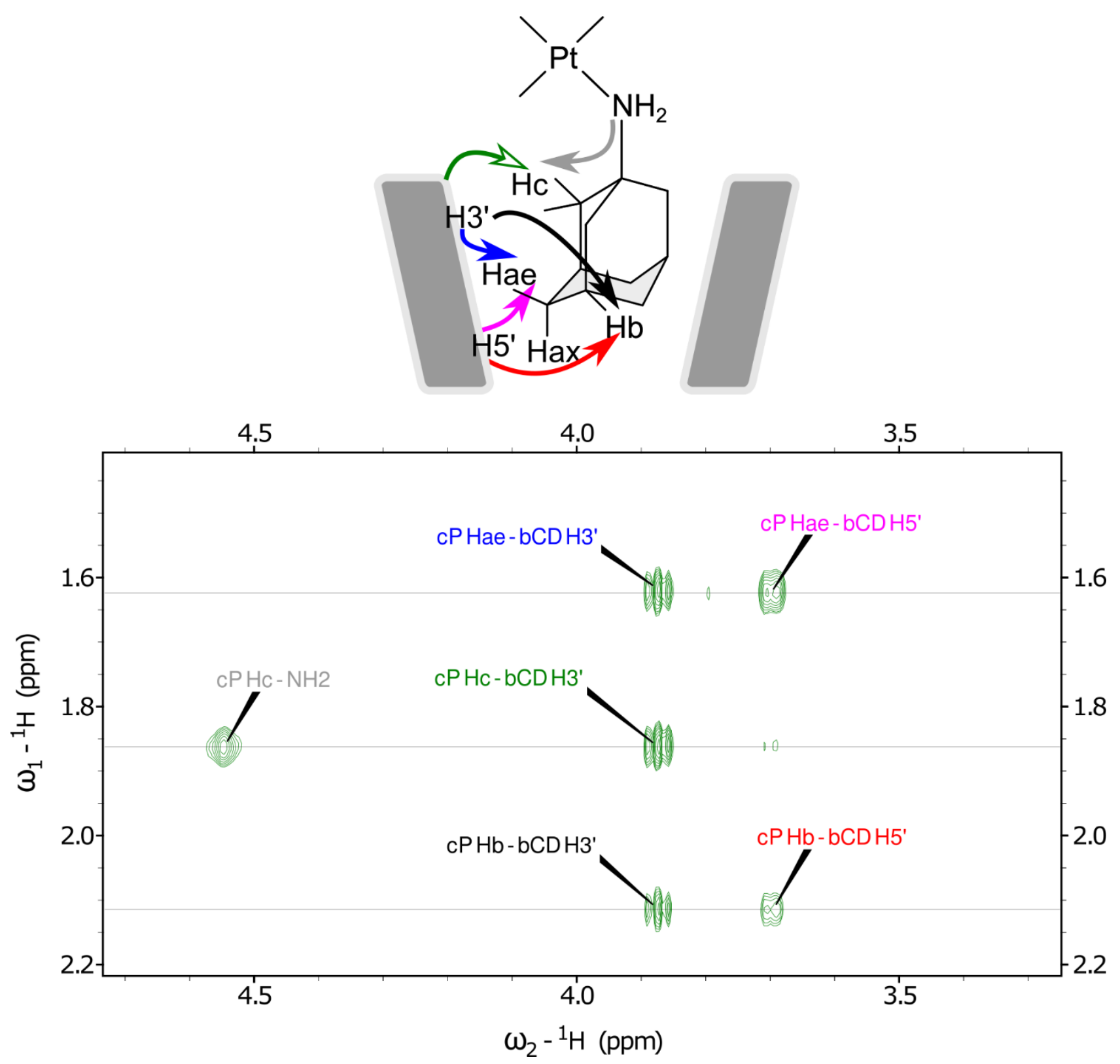

Figure S5a. ${ }^{1} \mathrm{H}-{ }^{1} \mathrm{H}$ ROESY $\left(t_{\mathrm{SL}}=75 \mathrm{~ms}\right)$ spectrum of 1a (labeled as $\left.\mathrm{cP}\right)$ interacting with $\beta$-CD in $\mathrm{D}_{2} \mathrm{O}$ measured at $293 \mathrm{~K}$. 

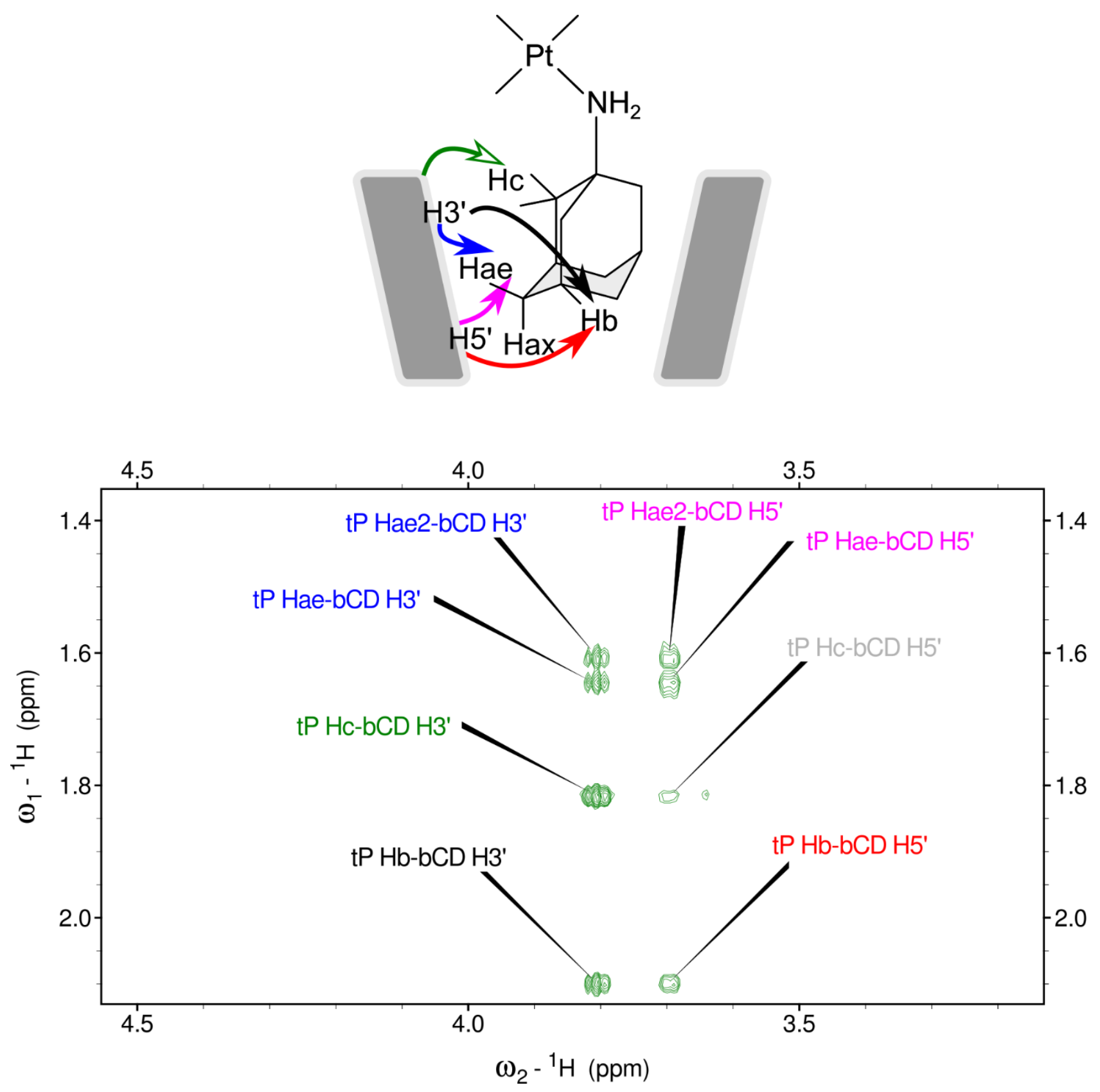

Figure S5b. ${ }^{1} \mathrm{H}-{ }^{1} \mathrm{H}$ ROESY $\left(t_{\mathrm{SL}}=75 \mathrm{~ms}\right)$ spectrum of $\mathbf{2 a}$ (labeled as $\left.\mathrm{tP}\right)$ interacting with $\beta$-CD in $\mathrm{D}_{2} \mathrm{O}$ measured at $293 \mathrm{~K}$. The intermolecular contacts shown indicate a greater preference of $\mathbf{2 a}$ for the wider secondary portal of $\beta$-CD. ${ }^{3-5}$ 


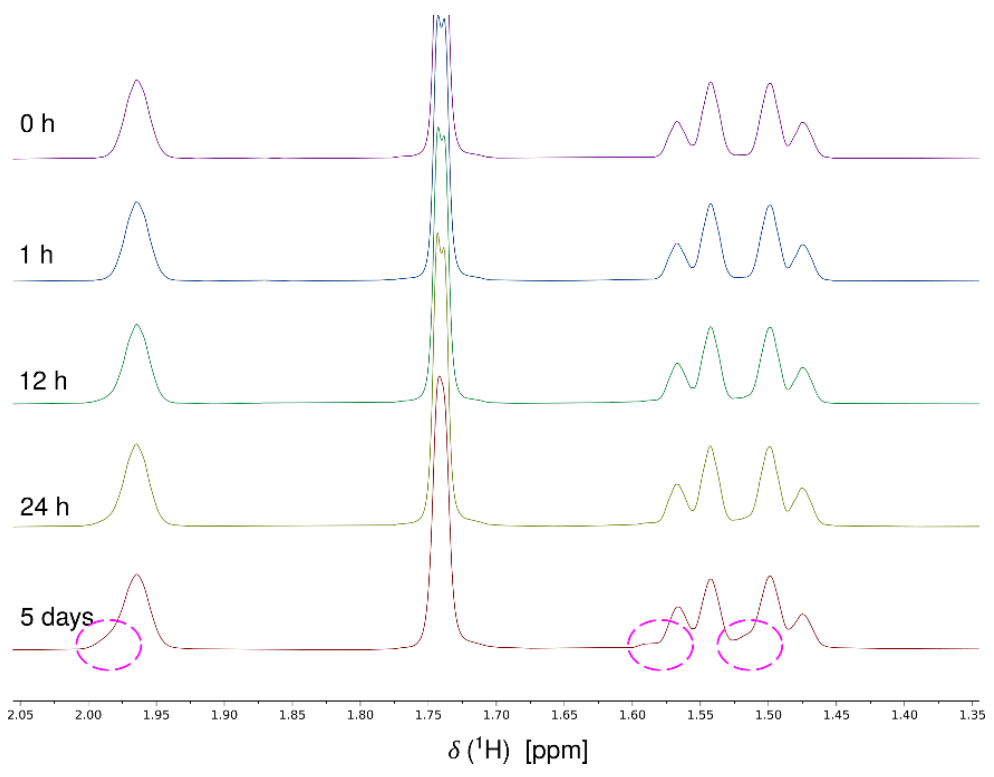

Figure S6. ${ }^{1} \mathrm{H}$ NMR spectra (adamantane region) of free $1 \mathbf{a}$ in $\mathrm{D}_{2} \mathrm{O}$ at $293 \mathrm{~K}$. Note the humps in the signals of $\mathrm{Hb}, \mathrm{Hax}$, and Hae appearing after 5 days. 
a) $1 \mathrm{a} @ \mathrm{CB} 7$ in $\mathrm{D}_{2} \mathrm{O}$
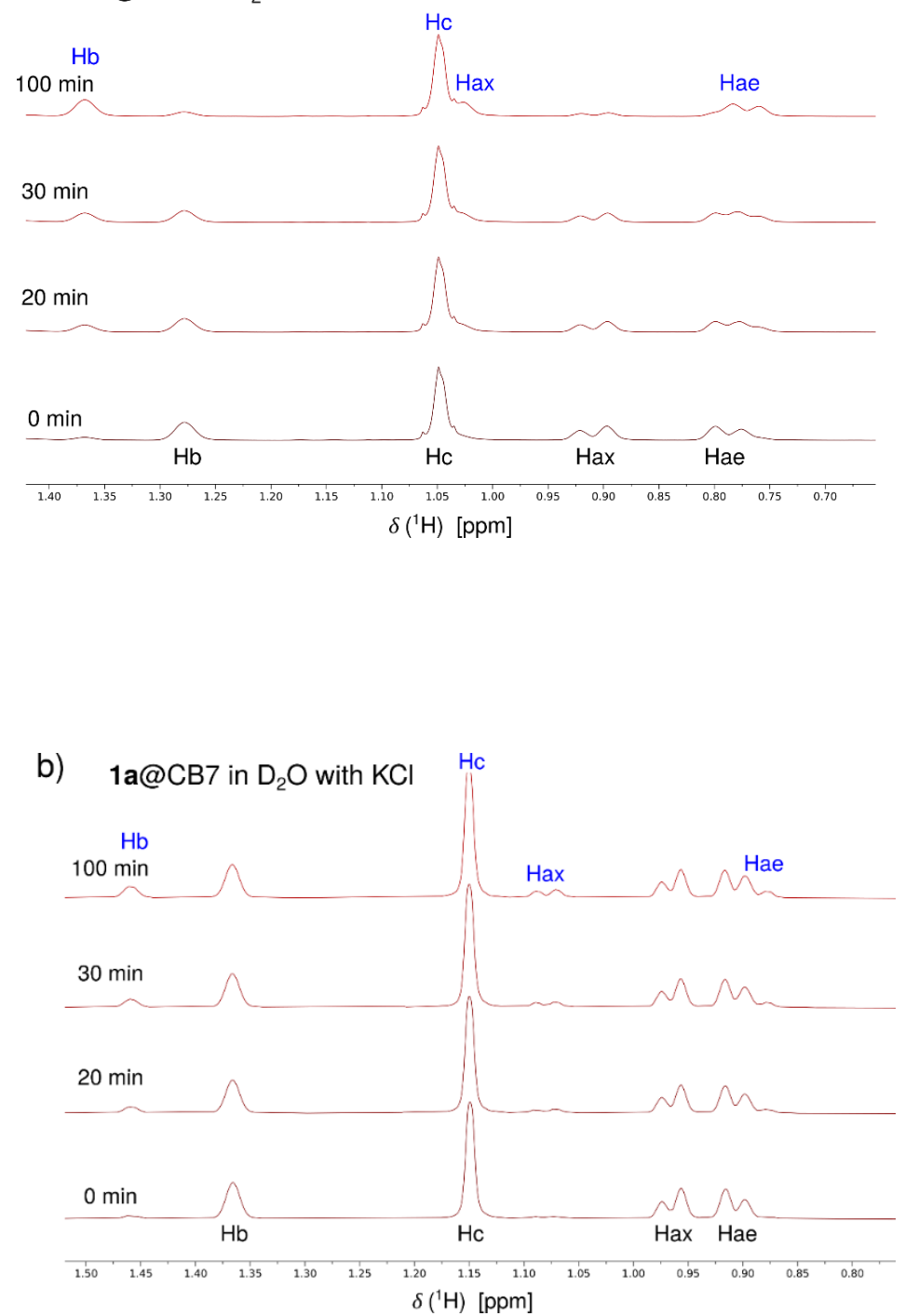

Figure S7. ${ }^{1} \mathrm{H}$ NMR spectra (adamantane region) of (a) 1a bound to $\mathrm{CB} 7$ in pure $\mathrm{D}_{2} \mathrm{O}$ and (b) $1 \mathrm{a}$ bound to $\mathrm{CB} 7 \mathrm{in} \sim 50 \mathrm{mM} \mathrm{KCl}$ solution measured at $293 \mathrm{~K}$. The presence of $\mathrm{Cl}^{-}$anions slows down the rate of the aquation process and reduces the separation of the Hax signals. 
Table S5. ${ }^{1} \mathrm{H}$ NMR shifts measured for compounds $1 \mathbf{a}$ and 1a_aq in the free and encapsulated form at $298 \mathrm{~K}$. The NMR shifts were referenced relative to the signal of dimethylsulphone ( $\delta=3.00 \mathrm{ppm})$.

\begin{tabular}{ccccccccc}
\hline & $\mathbf{1 a}$ & $\mathbf{1 a} \_\mathbf{a q}$ & $\boldsymbol{\Delta a q}^{\mathrm{a}}$ & $\mathbf{1 a} @ \mathbf{C B} 7^{\mathrm{b}}$ & $\Delta_{\mathrm{CB}} 7^{\mathrm{c}}$ & $\mathbf{1 a} \_\mathbf{a q} @ \mathbf{C B 7}$ & $\Delta \mathbf{A q C B}^{\mathrm{d}}$ & $\Delta^{\mathrm{e}}$ \\
$\mathrm{Hax}$ & 1.55 & 1.57 & $\mathbf{+ 0 . 0 2}$ & 0.89 & $\mathbf{- 0 . 6 6}$ & 1.04 & $\mathbf{- 0 . 5 3}$ & $\mathbf{+ 0 . 1 3}$ \\
$\mathrm{Hae}$ & 1.48 & 1.49 & $\mathbf{+ 0 . 0 1}$ & 0.80 & $\mathbf{- 0 . 6 8}$ & 0.77 & $\mathbf{- 0 . 7 2}$ & $\mathbf{- 0 . 0 4}$ \\
$\mathrm{Hb}$ & 1.96 & 1.98 & $\mathbf{+ 0 . 0 2}$ & 1.28 & $\mathbf{- 0 . 6 8}$ & 1.37 & $\mathbf{- 0 . 6 1}$ & $\mathbf{+ 0 . 0 7}$ \\
$\mathrm{Hc}$ & 1.73 & 1.73 & $\mathbf{0 . 0 0}$ & 1.05 & $\mathbf{- 0 . 6 8}$ & 1.05 & $\mathbf{- 0 . 6 8}$ & $\mathbf{0 . 0 0}$ \\
\hline
\end{tabular}

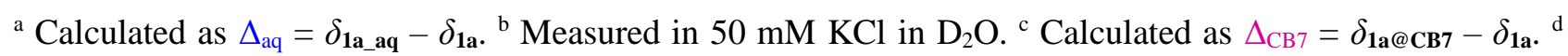
Calculated as $\Delta \mathrm{aq}_{\mathrm{CB} 7}=\delta_{\mathbf{1} \_\_a q} @ \mathbf{C B} 7-\delta_{\mathbf{1} \mathbf{a} \_ \text {aq. }}{ }^{\mathrm{e}} \Delta=\Delta \mathrm{aq}_{\mathrm{CB} 7}-\Delta_{\mathrm{CB} 7}$.
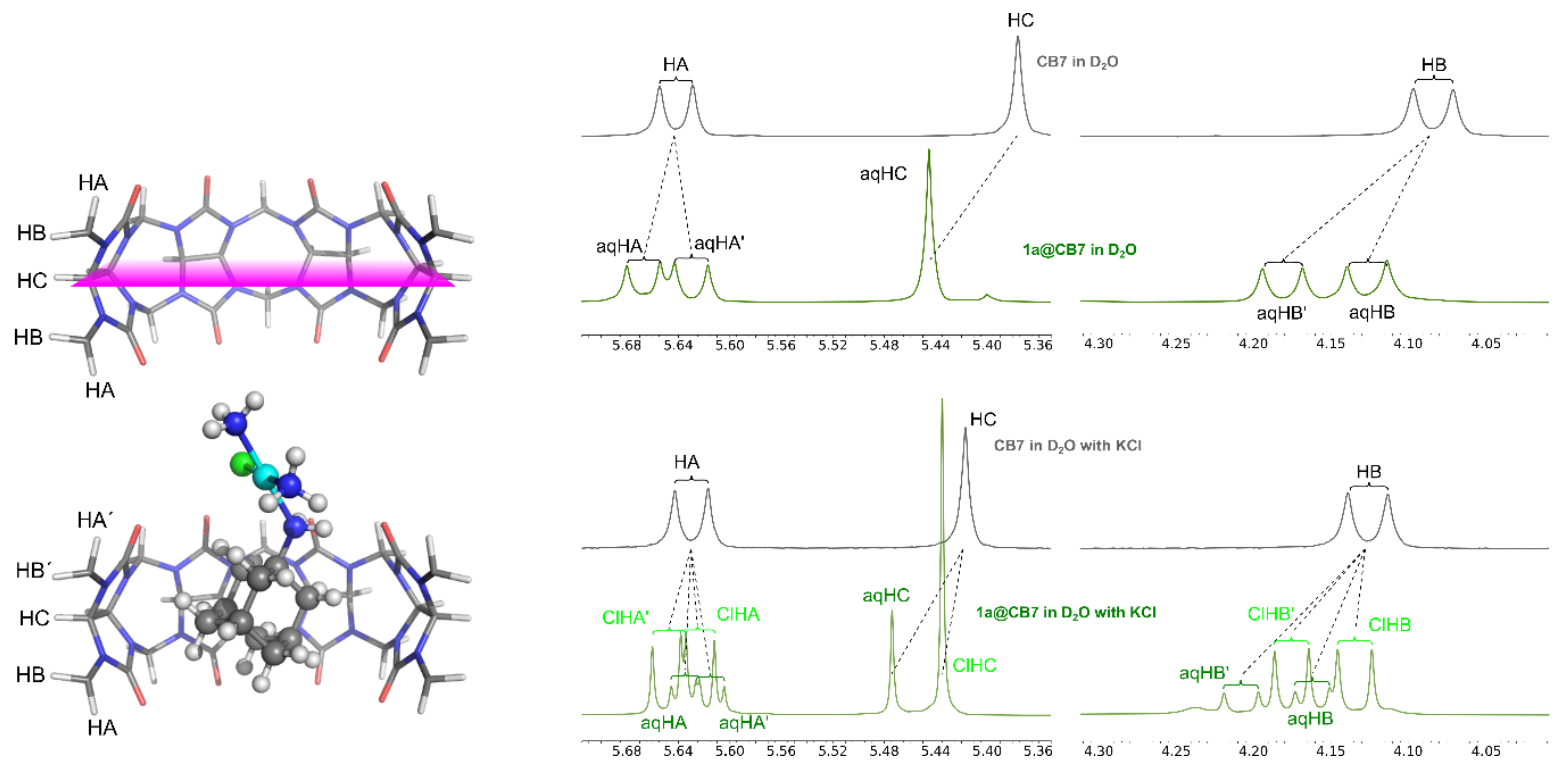

Figure S8. Detailed view of the splitting of the ${ }^{1} \mathrm{H}$ NMR signals of CB7 observed upon the complexation of 1a_aq in pure $\mathrm{D}_{2} \mathrm{O}$ (top) and $\mathbf{1 a}$ in $50 \mathrm{mM}$ aq. $\mathrm{KCl}$ (bottom). Note the presence of both $\mathrm{Cl}(\mathbf{1 a})$ and $\mathrm{H}_{2} \mathrm{O}$ (1a_aq) forms in the latter spectrum, HC, HA, and both HBs are more deshielded in the presence of 1a_aq. Only the closest proton HA' is shielded. The signal splitting which results from the loss of symmetry of the free CB7 (top structure with a schematic representation of the symmetry plane in magenta) indicates the formation of a host-guest complex with an unsymmetric positioning of the guest (bottom structure). 
Table S6. ${ }^{1} \mathrm{H}$ NMR shifts measured for CB7 signals in the free and bound form at $298 \mathrm{~K}$. The NMR shifts were referenced relative to the signal of dimethylsulphone $(\delta=3.00 \mathrm{ppm})$.

\begin{tabular}{|c|c|c|c|c|c|c|}
\hline & CB7 & 1a@CB7 / 1a_aq@CB7 & $\Delta(\mathbf{1 a}) / \Delta\left(\mathbf{1 a} \_\mathbf{a q}\right)$ & CB7 & 1a_aq@CB7 & $\Delta\left(1 \mathbf{a} \_\mathbf{a q}\right)$ \\
\hline & $\mathrm{D}_{2} \mathrm{O}(\mathrm{KCl})^{\mathrm{a}}$ & $\mathrm{D}_{2} \mathrm{O}(\mathrm{KCl})^{\mathrm{a}}$ & $\mathrm{D}_{2} \mathrm{O}(\mathrm{KCl})^{\mathrm{a}}$ & $\mathrm{D}_{2} \mathrm{O}$ & $\mathrm{D}_{2} \mathrm{O}$ & $\mathrm{D}_{2} \mathrm{O}$ \\
\hline HA & 5.64 & $5.63 / 5.64$ & $-0.01 / 0.00$ & 5.66 & 5.67 & +0.01 \\
\hline HA $^{\prime}$ & 5.64 & $5.65 / 5.62$ & $+0.01 /-0.02$ & 5.66 & 5.64 & -0.02 \\
\hline $\mathrm{HB}$ & 4.13 & $4.13 / 4.16$ & $\mathbf{0 . 0 0} /+\mathbf{0 . 0 3}$ & 4.09 & 4.13 & +0.04 \\
\hline $\mathrm{HB}^{\prime}$ & 4.13 & $4.17 / 4.21$ & $+0.04 /+0.08$ & 4.09 & 4.18 & +0.09 \\
\hline $\mathrm{HC}$ & 5.43 & $5.44 / 5.48$ & $+0.01 /+0.05$ & 5.39 & 5.45 & +0.06 \\
\hline
\end{tabular}

${ }^{\mathrm{a}}$ Measured in $50 \mathrm{mM} \mathrm{KCl}$ in $\mathrm{D}_{2} \mathrm{O}$.
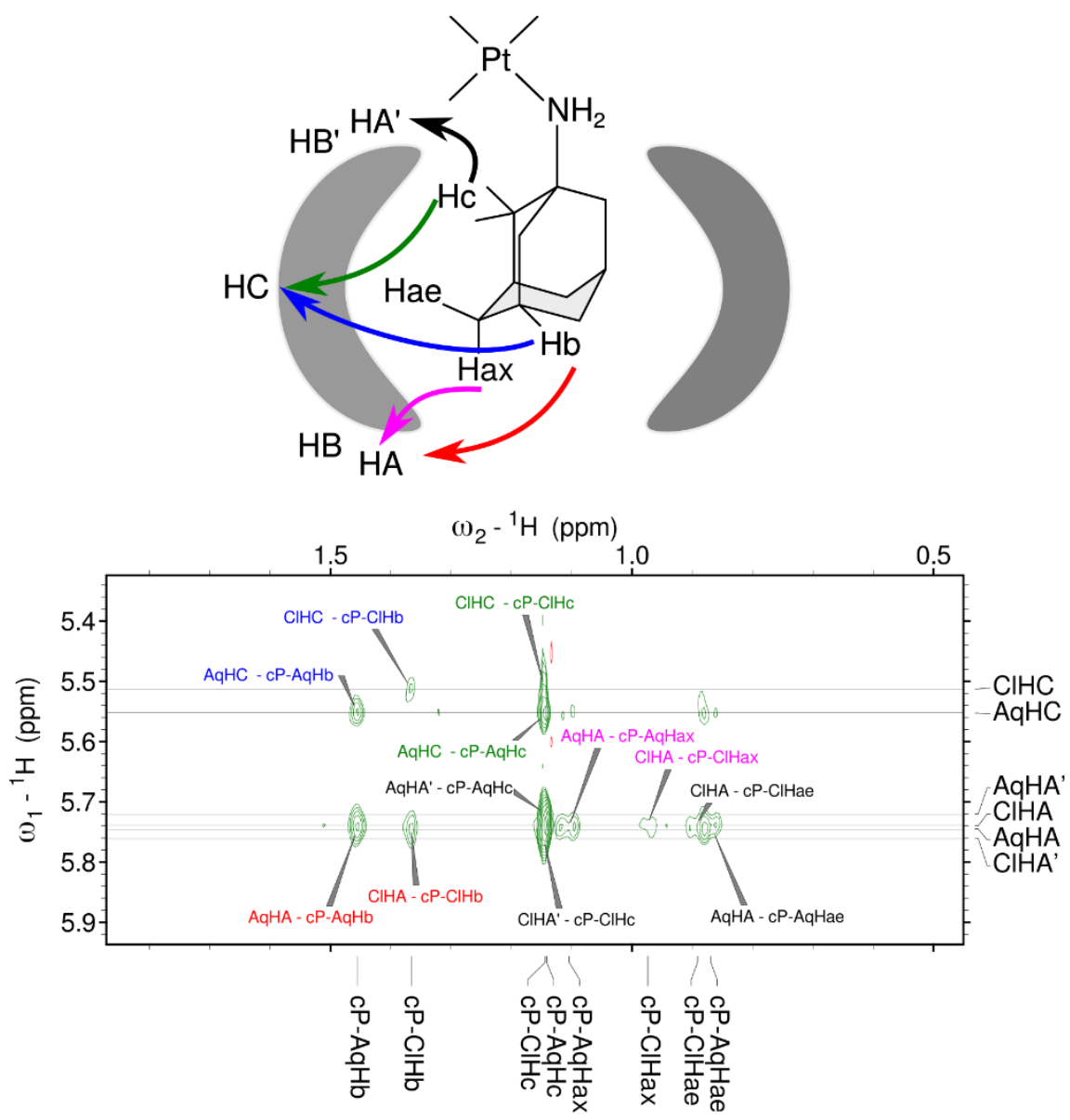

Figure S9. ${ }^{1} \mathrm{H}-{ }^{1} \mathrm{H}$ ROESY $\left(t_{\mathrm{SL}}=150 \mathrm{~ms}\right)$ spectrum of $1 \mathrm{a}\left(\right.$ labeled as $\mathrm{cP}$ ) bound to $\mathrm{CB} 7$ in $\mathrm{D}_{2} \mathrm{O}$ with $50 \mathrm{mM}$ $\mathrm{KCl}$ measured at $293 \mathrm{~K}$ showing weak intermolecular contacts between the Ad moiety and $\mathrm{CB} 7 . \mathrm{Both} \mathrm{Cl}$ and hydrolyzed forms (labeled as $\mathbf{C l}$ and aq, respectively) were identified, and individual signals of CB7 were unambiguously assigned. 
Table S7. The values of IC50 concentration were determined by MTT assay on the two cell lines A2780 and A2780/CP. The values are stated in $\mu \mathrm{M}$ concentration for 24 to 48 hours with the standard deviation.

\begin{tabular}{|c|c|c|}
\hline \multicolumn{3}{|c|}{$\mathbf{A 2 7 8 0}$} \\
\hline Compound & $24 \mathrm{~h}$ & $48 \mathrm{~h}$ \\
\hline cisPt & $16.5 \pm 5.0$ & $7.95 \pm 0.65$ \\
\hline cisPt@CB7 & $26.0 \pm 2.5$ & $17.2 \pm 3.8$ \\
\hline cisPt@ $\beta-C D$ & $22.3 \pm 4.6$ & $7.26 \pm 0.48$ \\
\hline $1 \mathrm{a}$ & $126 \pm 23$ & $82.1 \pm 8.2$ \\
\hline 1a@CB7 & $230 \pm 63$ & $218 \pm 41$ \\
\hline $1 \mathrm{1a} @ \beta-C D$ & $205 \pm 47$ & $102 \pm 20$ \\
\hline \multicolumn{3}{|c|}{$\mathrm{A} 2780 / \mathrm{CP}$} \\
\hline Compound & $24 \mathrm{~h}$ & $48 \mathrm{~h}$ \\
\hline cisPt & $52.0 \pm 5.8$ & $25.5 \pm 1.5$ \\
\hline cisPt@CB7 & $50.6 \pm 5.1$ & $43.8 \pm 5.9$ \\
\hline cisPt@ $\beta-C D$ & $41.8 \pm 5.7$ & $25.4 \pm 1.1$ \\
\hline $1 \mathrm{a}$ & $175 \pm 23$ & $112 \pm 15$ \\
\hline 1a@CB7 & $510 \pm 150$ & $457 \pm 37$ \\
\hline $1 \mathrm{a} @ \beta-C D$ & $320 \pm 100$ & $271 \pm 31$ \\
\hline
\end{tabular}


Table S8. The values of platinum content in the respective cell samples upon treatment of the two cell lines A2780 and A2780/CP. The values are stated in nanograms in $10^{9}$ cells with the standard deviation.

\begin{tabular}{lcc}
\hline & A2780 & \\
Compound & $\mathbf{2 4} \mathbf{h}$ & $\mathbf{4 8 ~ h}$ \\
\hline control & $79 \pm 15$ & $27.6 \pm 2.3$ \\
cisPt & $3200 \pm 130$ & $1511 \pm 82$ \\
cisPt $@ \mathbf{C B 7}$ & $1265 \pm 61$ & $1085 \pm 29$ \\
cisPt $@ \beta$-CD & $1099 \pm 18$ & $1282 \pm 24$ \\
$\mathbf{1 a}$ & $1425 \pm 37$ & $1867 \pm 80$ \\
$\mathbf{1 a} @ \mathbf{C B 7}$ & $504 \pm 50$ & $914 \pm 58$ \\
$\mathbf{1 a} @ \beta$-CD & $1268 \pm 75$ & $2263 \pm 75$ \\
\hline & $\mathbf{A 2 7 8 0 / C P}$ & \\
\hline Compound & $\mathbf{2 4} \mathbf{h}$ & $\mathbf{4 8 ~ h}$ \\
\hline control & $200 \pm 30$ & $29.1 \pm 8.9$ \\
cisPt & $909 \pm 65$ & $920 \pm 210$ \\
cisPt@CB7 & $599 \pm 69$ & $609 \pm 34$ \\
cisPt@ $\boldsymbol{\beta}$-CD & $627 \pm 38$ & $935 \pm 69$ \\
$\mathbf{1 a}$ & $955 \pm 46$ & $1043 \pm 43$ \\
$\mathbf{1 a} @ \mathbf{C B 7}$ & $304 \pm 19$ & $313 \pm 25$ \\
$\mathbf{1 a} @ \boldsymbol{\beta}$-CD & $962 \pm 64$ & $983 \pm 48$ \\
\hline
\end{tabular}


Table S9. DFT-calculated ${ }^{195} \mathrm{Pt}$ nuclear shielding constants and ${ }^{195} \mathrm{Pt}$ NMR shifts (in ppm) for compounds 1a and 2a, their hydrolyzed forms 1a_aq and 2a_aq, and their host-guest complexes with CB7 in water solution. ${ }^{a}$

\begin{tabular}{|c|c|c|c|c|}
\hline \multirow[b]{2}{*}{ Compound } & \multicolumn{2}{|c|}{$\begin{array}{c}\text { Two-component } \\
(2 \mathrm{c}-\mathrm{ZORA}, \text { PBE0 })^{\mathrm{a}}\end{array}$} & \multicolumn{2}{|c|}{$\begin{array}{l}\text { Four-component } \\
(4 c-D K S, \text { PBE })^{b}\end{array}$} \\
\hline & $\begin{array}{l}\text { Shielding } \\
\text { constant }(\sigma)\end{array}$ & $\begin{array}{l}\text { Chemical shift } \\
(\delta)^{\mathrm{c}}\end{array}$ & $\begin{array}{c}\text { Shielding } \\
\text { constant }(\sigma)\end{array}$ & $\begin{array}{l}\text { Chemical shift } \\
(\delta)^{\mathrm{c}}\end{array}$ \\
\hline cisplatin & 4139 & & 5741 & --- \\
\hline $\mathrm{PtCl}^{2-}$ & 1413 & & 3994 & --- \\
\hline $1 \mathbf{a}$ & 4447 & -2447 & 5914 & -2312 \\
\hline $\mathbf{2 a}$ & 4494 & -2494 & 6041 & -2439 \\
\hline 1a_aq & 4156 & -2157 & 5558 & -1956 \\
\hline 2a_aq & 4182 & -2183 & 5582 & -1980 \\
\hline 1a@CB7 & 4503 & -2503 & 6026 & -2424 \\
\hline 2a@CB7 & 4544 & -2545 & 6068 & -2466 \\
\hline 1a_aq@CB7 & 4154 & -2155 & 5624 & -2022 \\
\hline 2a_aq@CB7 & 4217 & -2218 & 5664 & -2062 \\
\hline
\end{tabular}

a 2c-ZORA results calculated with QZ4P, TZ2P basis sets and using the implicit COSMO model for solvent.

${ }^{\mathrm{b}} 4 \mathrm{c}-\mathrm{DKS}$ results calculated with uncontracted dyall_vtz (Pt), pcS-2 (Cl, $\left.\mathrm{H}_{2} \mathrm{O}, \mathrm{NH}_{2}, \mathrm{NH}_{3}\right)$, IGLO-II (adamantyl, CB[7]) basis sets, and including the PCM model for solvent.

${ }^{c}$ The NMR shifts were calculated using the experimental NMR shift of cisplatin $(\delta=-2139$ ppm $)$ as secondary reference, and are reported relative to $\mathrm{PtCl}_{6}{ }^{2-}$. 


\section{REFERENCES}

(1) Appleton, T. G.; Bailey, A. J.; Barnham, K. J.; Hall, J. R. Aspects of the Solution Chemistry of Trans-Diammineplatinum(II) Complexes. Inorg. Chem. 1992, 31 (14), 3077-3082. https://doi.org/10.1021/ic00040a017.

(2) Frassineti, C.; Ghelli, S.; Gans, P.; Sabatini, A.; Moruzzi, M. S.; Vacca, A. Nuclear Magnetic Resonance as a Tool for Determining Protonation Constants of Natural Polyprotic Bases in Solution. Analytical Biochemistry 1995, 231 (2), 374-382. https://doi.org/10.1006/abio.1995.9984.

(3) Branná, P.; Černochová, J.; Rouchal, M.; Kulhánek, P.; Babinský, M.; Marek, R.; Nečas, M.; Kuřitka, I.; Vícha, R. Cooperative Binding of Cucurbit[n]Urils and $\beta$-Cyclodextrin to Heteroditopic Imidazolium-Based Guests. J. Org. Chem. 2016, 81 (20), 9595-9604. https://doi.org/10.1021/acs.joc.6b01564.

(4) Malali, S.; Chyba, J.; Knor, M.; Horní, M.; Nečas, M.; Novotný, J.; Marek, R. Zwitterionic $\mathrm{Ru}(\mathrm{III})$ Complexes: Stability of Metal-Ligand Bond and Host-Guest Binding with Cucurbit[7]Uril. Inorg. Chem. 2020, 59 (14), 10185-10196. https://doi.org/10.1021/acs.inorgchem.0c01328.

(5) Tomeček, J.; Čablová, A.; Hromádková, A.; Novotný, J.; Marek, R.; Durník, I.; Kulhánek, P.; Prucková, Z.; Rouchal, M.; Dastychová, L.; Vícha, R. Modes of Micromolar Host-Guest Binding of $\beta$-Cyclodextrin Complexes Revealed by NMR Spectroscopy in Salt Water. J. Org. Chem. 2021, 86 (6), 4483-4496. https://doi.org/10.1021/acs.joc.0c02917. 


\section{CARTESIAN COORDINATES}

$\begin{array}{lrrr}\text { Compound 1a } & & \\ \text { 38 } & & & \\ \mathrm{Pt} & 10.988962000 & 10.567856000 & 9.945600000 \\ \mathrm{Cl} & 10.665116000 & 11.646644000 & 11.967031000 \\ \mathrm{~N} & 12.272321000 & 9.259574000 & 10.890348000 \\ \mathrm{~N} & 9.717100000 & 11.934411000 & 9.045749000 \\ \mathrm{C} & 8.269037000 & 11.601938000 & 8.794922000 \\ \mathrm{C} & 7.566686000 & 11.299381000 & 10.123168000 \\ \mathrm{C} & 8.158733000 & 10.386055000 & 7.868509000 \\ \mathrm{C} & 7.607649000 & 12.819343000 & 8.126836000 \\ \mathrm{C} & 6.082437000 & 10.996328000 & 9.856094000 \\ \mathrm{C} & 6.675185000 & 10.083283000 & 7.593392000 \\ \mathrm{C} & 6.123267000 & 12.517357000 & 7.858932000 \\ \mathrm{C} & 5.970581000 & 9.777535000 & 8.925444000 \\ \mathrm{C} & 6.015361000 & 11.300450000 & 6.925973000 \\ \mathrm{C} & 5.419255000 & 12.213874000 & 9.191513000 \\ \mathrm{~N} & 11.378337000 & 9.627142000 & 8.144063000 \\ \mathrm{H} & 11.946273000 & 8.290508000 & 10.845807000 \\ \mathrm{H} & 12.343022000 & 9.509557000 & 11.881322000 \\ \mathrm{H} & 13.219045000 & 9.286965000 & 10.502965000 \\ \mathrm{H} & 12.276237000 & 9.938924000 & 7.763444000 \\ \mathrm{H} & 10.668710000 & 9.814549000 & 7.431238000 \\ \mathrm{H} & 11.427110000 & 8.609411000 & 8.240551000 \\ \mathrm{H} & 10.127526000 & 12.245027000 & 8.156571000 \\ \mathrm{H} & 9.739967000 & 12.762351000 & 9.655338000 \\ \mathrm{H} & 7.667411000 & 12.161625000 & 10.800751000 \\ \mathrm{H} & 8.058138000 & 10.441945000 & 10.607205000 \\ \mathrm{H} & 8.680916000 & 10.588851000 & 6.917769000 \\ \mathrm{H} & 7.705965000 & 13.697412000 & 8.784906000 \\ \mathrm{H} & 8.126333000 & 13.049206000 & 7.182003000 \\ \mathrm{H} & 5.661704000 & 13.395154000 & 7.383068000 \\ \mathrm{H} & 6.427367000 & 8.895155000 & 9.400747000 \\ \mathrm{H} & 4.911205000 & 9.541064000 & 8.741800000 \\ \mathrm{H} & 4.352417000 & 12.008844000 & 9.012849000 \\ \mathrm{H} & 5.479024000 & 13.088457000 & 9.858300000 \\ \mathrm{H} & 5.587529000 & 10.779378000 & 10.814232000 \\ \mathrm{H} & 6.505085000 & 11.516842000 & 5.963358000 \\ \mathrm{H} & 4.957237000 & 11.082329000 & 6.714683000 \\ \mathrm{H} & 6.610468000 & 9.210567000 & 6.927216000 \\ \mathrm{H} & 8.630295000 & 9.516208000 & 8.351283000\end{array}$

\section{Compound 1a@CB7}

$\begin{array}{lr}\text { 164 } & \\ \text { Pt } & 10.940863000 \\ \text { Cl } & 10.390575000 \\ \mathrm{~N} & 12.120385000 \\ \mathrm{~N} & 9.758537000 \\ \mathrm{C} & 8.346719000 \\ \mathrm{C} & 7.448822000 \\ \mathrm{C} & 8.287297000 \\ \mathrm{C} & 7.854680000 \\ \mathrm{C} & 5.998989000 \\ \mathrm{C} & 6.836903000 \\ \mathrm{C} & 6.405157000 \\ \mathrm{C} & 5.935975000 \\ \mathrm{C} & 6.350596000 \\ \mathrm{C} & 5.513770000 \\ \mathrm{O} & 9.295018000 \\ \mathrm{O} & 4.078492000 \\ \mathrm{~N} & 8.348552000 \\ \mathrm{~N} & 7.188688000 \\ \mathrm{~N} & 6.272010000 \\ & \end{array}$

10.139999000
10.555926000
8.576916000
11.730344000
11.495372000
11.180443000
10.334503000
12.778897000
10.999096000
10.143249000
12.593862000
9.833983000
11.426739000
12.288139000
13.977255000
16.037797000
15.823091000
14.801833000
16.662538000

10.162383000

12.369036000

10.817582000

9.557578000

9.073245000

10.276055000

8.074411000

8.380348000

9.798900000

7.594472000

7.903220000

8.800068000

6.904692000

9.117683000

11.785125000

9.281483000

10.729598000

12.342822000

9.746452000 


\begin{tabular}{|c|c|c|c|}
\hline $\mathrm{N}$ & 5.109753000 & 15.597506000 & 11.322342000 \\
\hline C & 8.372589000 & 14.778939000 & 11.634523000 \\
\hline $\mathrm{C}$ & 7.172271000 & 16.658972000 & 10.878858000 \\
\hline $\mathrm{C}$ & 6.352300000 & 15.932650000 & 11.989146000 \\
\hline $\mathrm{C}$ & 5.051222000 & 16.085075000 & 10.031317000 \\
\hline $\mathrm{C}$ & 9.537951000 & 16.260701000 & 10.033029000 \\
\hline $\mathrm{C}$ & 6.519413000 & 17.406115000 & 8.535479000 \\
\hline 0 & 11.098512000 & 14.306114000 & 8.579289000 \\
\hline 0 & 5.795476000 & 16.066896000 & 6.078634000 \\
\hline $\mathrm{N}$ & 10.233232000 & 15.259245000 & 6.641505000 \\
\hline $\mathrm{N}$ & 9.521807000 & 16.019053000 & 8.609985000 \\
\hline $\mathrm{N}$ & 8.078370000 & 15.883005000 & 5.661767000 \\
\hline $\mathrm{N}$ & 7.412739000 & 16.755836000 & 7.604153000 \\
\hline $\mathrm{C}$ & 10.357776000 & 15.106993000 & 8.005624000 \\
\hline $\mathrm{C}$ & 9.317767000 & 16.320149000 & 6.275985000 \\
\hline $\mathrm{C}$ & 8.853622000 & 16.885714000 & 7.654807000 \\
\hline $\mathrm{C}$ & 6.968629000 & 16.210587000 & 6.415777000 \\
\hline $\mathrm{C}$ & 11.078137000 & 14.567352000 & 5.699816000 \\
\hline $\mathrm{C}$ & 7.974338000 & 15.485537000 & 4.277226000 \\
\hline 0 & 11.416029000 & 11.769476000 & 6.248849000 \\
\hline 0 & 6.118814000 & 13.380499000 & 3.575163000 \\
\hline $\mathrm{N}$ & 10.051592000 & 11.354797000 & 4.401081000 \\
\hline $\mathrm{N}$ & 10.438207000 & 13.465897000 & 5.014454000 \\
\hline $\mathrm{N}$ & 7.985142000 & 12.023809000 & 3.265821000 \\
\hline $\mathrm{N}$ & 8.287266000 & 14.096322000 & 4.032527000 \\
\hline $\mathrm{C}$ & 10.700006000 & 12.153297000 & 5.318336000 \\
\hline $\mathrm{C}$ & 9.421563000 & 12.142834000 & 3.347703000 \\
\hline $\mathrm{C}$ & 9.637146000 & 13.614223000 & 3.817570000 \\
\hline $\mathrm{C}$ & 7.330241000 & 13.185671000 & 3.627234000 \\
\hline $\mathrm{C}$ & 10.377609000 & 9.953562000 & 4.228183000 \\
\hline $\mathrm{C}$ & 7.313777000 & 10.918667000 & 2.625647000 \\
\hline 0 & 10.254156000 & 8.003448000 & 6.369175000 \\
\hline 0 & 4.896347000 & 9.851319000 & 3.796729000 \\
\hline $\mathrm{N}$ & 8.289801000 & 7.254397000 & 5.388369000 \\
\hline $\mathrm{N}$ & 9.288636000 & 9.051706000 & 4.524048000 \\
\hline $\mathrm{N}$ & 6.169103000 & 7.997993000 & 4.402114000 \\
\hline $\mathrm{N}$ & 7.194879000 & 9.735653000 & 3.446337000 \\
\hline $\mathrm{C}$ & 9.363738000 & 8.100940000 & 5.521138000 \\
\hline $\mathrm{C}$ & 7.518891000 & 7.516814000 & 4.189025000 \\
\hline $\mathrm{C}$ & 8.242531000 & 8.746406000 & 3.559537000 \\
\hline $\mathrm{C}$ & 5.970916000 & 9.262978000 & 3.881571000 \\
\hline $\mathrm{C}$ & 8.111315000 & 6.087573000 & 6.224785000 \\
\hline $\mathrm{C}$ & 5.064709000 & 7.120485000 & 4.718505000 \\
\hline 0 & 8.293471000 & 6.553985000 & 9.078544000 \\
\hline 0 & 2.963740000 & 8.095441000 & 6.441070000 \\
\hline $\mathrm{N}$ & 5.997802000 & 6.158352000 & 9.148673000 \\
\hline $\mathrm{N}$ & 7.020174000 & 6.184918000 & 7.164158000 \\
\hline $\mathrm{N}$ & 3.858578000 & 6.714275000 & 8.087309000 \\
\hline $\mathrm{N}$ & 4.907019000 & 6.853771000 & 6.126281000 \\
\hline $\mathrm{C}$ & 7.219545000 & 6.331909000 & 8.525505000 \\
\hline $\mathrm{C}$ & 4.948771000 & 5.771606000 & 8.223693000 \\
\hline $\mathrm{C}$ & 5.655701000 & 5.833217000 & 6.834139000 \\
\hline $\mathrm{C}$ & 3.822626000 & 7.313895000 & 6.845567000 \\
\hline $\mathrm{C}$ & 5.874835000 & 6.018263000 & 10.581316000 \\
\hline $\mathrm{C}$ & 2.771824000 & 6.824293000 & 9.031470000 \\
\hline 0 & 7.044970000 & 7.932316000 & 12.394627000 \\
\hline 0 & 1.859400000 & 9.533330000 & 9.506465000 \\
\hline $\mathrm{N}$ & 4.871764000 & 8.647936000 & 12.827154000 \\
\hline $\mathrm{N}$ & 5.196115000 & 7.113893000 & 11.236531000 \\
\hline $\mathrm{N}$ & 2.880011000 & 9.366060000 & 11.593134000 \\
\hline $\mathrm{N}$ & 3.049931000 & 7.650574000 & 10.180287000 \\
\hline $\mathrm{C}$ & 5.838353000 & 7.908967000 & 12.171486000 \\
\hline $\mathrm{C}$ & 3.533025000 & 8.335684000 & 12.384522000 \\
\hline $\mathrm{C}$ & 3.748985000 & 7.184471000 & 11.355439000 \\
\hline $\mathrm{C}$ & 2.533150000 & 8.920340000 & 10.332652000 \\
\hline $\mathrm{C}$ & 5.161094000 & 9.495137000 & 13.961387000 \\
\hline $\mathrm{C}$ & 2.277806000 & 10.541657000 & 12.176143000 \\
\hline 0 & 7.479491000 & 11.210517000 & 13.845420000 \\
\hline
\end{tabular}




\begin{tabular}{|c|c|c|c|}
\hline 0 & 2.358740000 & 13.061792000 & 10.770190000 \\
\hline $\mathrm{N}$ & 6.010540000 & 12.964991000 & 13.40850400 \\
\hline $\mathrm{N}$ & 5.179183000 & 10.908735000 & 13.66908300 \\
\hline $\mathrm{N}$ & 3.937471000 & 13.733723000 & 12.34323200 \\
\hline $\mathrm{N}$ & 3.202218000 & 11.628087000 & 12.40060300 \\
\hline C & 6.350391000 & 11.643119000 & 13.65364700 \\
\hline $\mathrm{C}$ & 4.579225000 & 13.186668000 & 13.5175840 \\
\hline $\mathrm{C}$ & 3.999503000 & 11.741014000 & 13.60925300 \\
\hline $\mathrm{C}$ & 3.093454000 & 12.830307000 & 11.7279450 \\
\hline $\mathrm{C}$ & 6.984800000 & 14.021593000 & 13.5441960 \\
\hline $\mathrm{C}$ & 3.933738000 & 15.141100000 & 12.0265730 \\
\hline $\mathrm{N}$ & 11.540458000 & 9.738268000 & 8.2301790 \\
\hline $\mathrm{H}$ & 11.813373000 & 7.674932000 & 10.4440920 \\
\hline $\mathrm{H}$ & 12.059919000 & 8.522166000 & 11.83911900 \\
\hline $\mathrm{H}$ & 13.109007000 & 8.690335000 & 10.5788430 \\
\hline $\mathrm{H}$ & 12.510758000 & 9.413307000 & 8.2165970 \\
\hline $\mathrm{H}$ & 11.502789000 & 10.551301000 & 7.5931370 \\
\hline $\mathrm{H}$ & 10.974033000 & 9.010860000 & 7.76400300 \\
\hline $\mathrm{H}$ & 10.264448000 & 12.275132000 & 8.8433350 \\
\hline $\mathrm{H}$ & 9.691272000 & 12.374634000 & 10.36327100 \\
\hline $\mathrm{H}$ & 7.512831000 & 12.000384000 & 11.00633600 \\
\hline $\mathrm{H}$ & 7.808023000 & 10.274194000 & 10.7847070 \\
\hline $\mathrm{H}$ & 8.939103000 & 10.547037000 & 7.2123720 \\
\hline $\mathrm{H}$ & 7.918872000 & 13.630336000 & 9.07404400 \\
\hline $\mathrm{H}$ & 8.505129000 & 13.004337000 & 7.5188290 \\
\hline $\mathrm{H}$ & 6.069903000 & 13.521485000 & 7.41498100 \\
\hline $\mathrm{H}$ & 6.260027000 & 8.904548000 & 9.28838400 \\
\hline $\mathrm{H}$ & 4.899012000 & 9.679434000 & 8.46329400 \\
\hline $\mathrm{H}$ & 4.465619000 & 12.174833000 & 8.80189900 \\
\hline $\mathrm{H}$ & 5.547114000 & 13.125522000 & 9.83181700 \\
\hline $\mathrm{H}$ & 5.362633000 & 10.777665000 & 10.66805000 \\
\hline $\mathrm{H}$ & 6.981961000 & 11.645110000 & 6.0294770 \\
\hline $\mathrm{H}$ & 5.323364000 & 11.288163000 & 6.53443300 \\
\hline $\mathrm{H}$ & 6.161259000 & 9.231222000 & 14.32299200 \\
\hline $\mathrm{H}$ & 4.414961000 & 9.297365000 & 14.74520000 \\
\hline $\mathrm{H}$ & 5.340032000 & 5.080521000 & 10.8010670 \\
\hline $\mathrm{H}$ & 6.889365000 & 5.973085000 & 10.99365700 \\
\hline $\mathrm{H}$ & 7.948014000 & 5.210649000 & 5.58091800 \\
\hline $\mathrm{H}$ & 9.035146000 & 5.960742000 & 6.80004000 \\
\hline $\mathrm{H}$ & 10.705067000 & 9.795041000 & 3.18863500 \\
\hline $\mathrm{H}$ & 11.200621000 & 9.713573000 & 4.91036100 \\
\hline $\mathrm{H}$ & 11.927007000 & 14.157532000 & 6.25753900 \\
\hline $\mathrm{H}$ & 11.437529000 & 15.284824000 & 4.94865400 \\
\hline $\mathrm{H}$ & 9.675163000 & 17.338011000 & 10.21160100 \\
\hline $\mathrm{H}$ & 10.386699000 & 15.704702000 & 10.44672000 \\
\hline $\mathrm{H}$ & 7.938720000 & 13.541745000 & 13.79282100 \\
\hline $\mathrm{H}$ & 6.683356000 & 14.703664000 & 14.3553130 \\
\hline $\mathrm{H}$ & 5.203016000 & 6.168095000 & 4.18370800 \\
\hline $\mathrm{H}$ & 4.146981000 & 7.610527000 & 4.37409700 \\
\hline $\mathrm{H}$ & 1.924852000 & 7.273806000 & 8.5013810 \\
\hline $\mathrm{H}$ & 2.501193000 & 5.816575000 & 9.38014800 \\
\hline $\mathrm{H}$ & 1.807089000 & 10.267773000 & 13.13382400 \\
\hline $\mathrm{H}$ & 1.513971000 & 10.899603000 & 11.47634800 \\
\hline $\mathrm{H}$ & 3.072402000 & 15.332078000 & $11.3765740 \mathrm{C}$ \\
\hline $\mathrm{H}$ & 3.830655000 & 15.713837000 & 12.96121500 \\
\hline $\mathrm{H}$ & 5.554878000 & 17.535751000 & 8.03193900 \\
\hline $\mathrm{H}$ & 6.938837000 & 18.389057000 & 8.7973950 \\
\hline $\mathrm{H}$ & 8.644642000 & 16.115834000 & 3.67321100 \\
\hline $\mathrm{H}$ & 6.935320000 & 15.645068000 & 3.96741300 \\
\hline $\mathrm{H}$ & 6.298904000 & 11.250922000 & 2.38046400 \\
\hline $\mathrm{H}$ & 7.855260000 & 10.659057000 & 1.70317100 \\
\hline $\mathrm{H}$ & 8.651016000 & 9.415304000 & 8.55807200 \\
\hline $\mathrm{H}$ & 9.824312000 & 17.057907000 & 5.63685200 \\
\hline $\mathrm{H}$ & 9.137462000 & 17.934176000 & 7.8265040 \\
\hline $\mathrm{H}$ & 7.466826000 & 17.686284000 & 11.1397380 \\
\hline $\mathrm{H}$ & 6.155789000 & 16.552267000 & 12.8766830 \\
\hline $\mathrm{H}$ & 4.355367000 & 13.810678000 & 14.39663000 \\
\hline $\mathrm{H}$ & 3.367660000 & 11.571383000 & 14.49465600 \\
\hline
\end{tabular}




$\begin{array}{rr}\mathrm{H} & 2.901442000 \\ \mathrm{H} & 3.344878000 \\ \mathrm{H} & 4.560540000 \\ \mathrm{H} & 5.629783000 \\ \mathrm{H} & 7.508886000 \\ \mathrm{H} & 8.688062000 \\ \mathrm{H} & 9.885950000 \\ \mathrm{H} & 10.160803000 \\ \mathrm{H} & 6.805065000\end{array}$
8.056633000
6.213652000
4.775331000
13.241196000
11.679362000
8.483205000
4.884611000
6.626221000
6.277378000
8.543960000
3.543173000
11.911293000
14.246408000
2.574062000
2.378078000
3.085863000
9.302825000
6.885752000

\section{Compound 1a_aq@CB7}

\begin{tabular}{|c|c|c|c|}
\hline o & & & \\
\hline Pt & 10.109098000 & 9.908138000 & 10.204085000 \\
\hline $\mathrm{N}$ & 10.723933000 & 7.981859000 & 10.622095000 \\
\hline $\mathrm{N}$ & 9.303894000 & 11.749645000 & 9.674889000 \\
\hline $\mathrm{C}$ & 7.946960000 & 11.644488000 & 9.00978500 \\
\hline $\mathrm{C}$ & 6.891664000 & 11.360412000 & 10.08604400 \\
\hline $\mathrm{C}$ & 7.946935000 & 10.505202000 & 7.97657500 \\
\hline $\mathrm{C}$ & 7.602552000 & 12.961500000 & 8.29680100 \\
\hline $\mathrm{C}$ & 5.505076000 & 11.240836000 & 9.43926200 \\
\hline $\mathrm{C}$ & 6.558822000 & 10.383722000 & 7.33370400 \\
\hline $\mathrm{C}$ & 6.210991000 & 12.845386000 & 7.64607200 \\
\hline $\mathrm{C}$ & 5.518760000 & 10.089483000 & 8.42398700 \\
\hline $\mathrm{C}$ & 6.220596000 & 11.700016000 & 6.62305700 \\
\hline $\mathrm{C}$ & 5.161719000 & 12.557363000 & 8.72997500 \\
\hline 0 & 9.321849000 & 13.988665000 & 11.83032700 \\
\hline 0 & 4.166021000 & 15.984415000 & 9.32518800 \\
\hline $\mathrm{N}$ & 8.411882000 & 15.838650000 & 10.74681200 \\
\hline $\mathrm{N}$ & 7.303871000 & 14.958074000 & 12.46607500 \\
\hline $\mathrm{N}$ & 6.342272000 & 16.686412000 & 9.76422500 \\
\hline $\mathrm{N}$ & 5.223070000 & 15.609347000 & 11.36756000 \\
\hline $\mathrm{C}$ & 8.437308000 & 14.838339000 & 11.69405100 \\
\hline $\mathrm{C}$ & 7.255035000 & 16.708075000 & 10.88527400 \\
\hline $\mathrm{C}$ & 6.441349000 & 16.035571000 & 12.03189200 \\
\hline $\mathrm{C}$ & 5.139569000 & 16.077374000 & 10.06768300 \\
\hline $\mathrm{C}$ & 9.603571000 & 16.238084000 & 10.03289100 \\
\hline $\mathrm{C}$ & 6.576745000 & 17.398845000 & 8.52906100 \\
\hline 0 & 11.026397000 & 14.170622000 & 8.60628700 \\
\hline 0 & 5.856377000 & 16.122291000 & 6.03381700 \\
\hline $\mathrm{N}$ & 10.260585000 & 15.192985000 & 6.66097200 \\
\hline $\mathrm{N}$ & 9.561979000 & 15.980051000 & 8.61804900 \\
\hline $\mathrm{N}$ & 8.139280000 & 15.874171000 & 5.65084100 \\
\hline $\mathrm{N}$ & 7.466571000 & 16.732247000 & 7.60148300 \\
\hline $\mathrm{C}$ & 10.350624000 & 15.022291000 & 8.02363900 \\
\hline $\mathrm{C}$ & 9.380154000 & 16.279534000 & 6.28514100 \\
\hline $\mathrm{C}$ & 8.910706000 & 16.852003000 & 7.65919700 \\
\hline $\mathrm{C}$ & 7.027438000 & 16.225567000 & 6.39212000 \\
\hline $\mathrm{C}$ & 11.127342000 & 14.506021000 & 5.73772800 \\
\hline $\mathrm{C}$ & 8.050048000 & 15.473097000 & 4.26404300 \\
\hline 0 & 11.432804000 & 11.688016000 & 6.25164400 \\
\hline 0 & 6.203755000 & 13.378915000 & 3.50187900 \\
\hline $\mathrm{N}$ & 10.145040000 & 11.331673000 & 4.34379700 \\
\hline $\mathrm{N}$ & 10.495477000 & 13.422520000 & 5.02509900 \\
\hline $\mathrm{N}$ & 8.069695000 & 12.011109000 & 3.23552600 \\
\hline $\mathrm{N}$ & 8.363321000 & 14.081381000 & 4.02066500 \\
\hline $\mathrm{C}$ & 10.753490000 & 12.104510000 & 5.30779200 \\
\hline $\mathrm{C}$ & 9.508143000 & 12.137313000 & 3.31323400 \\
\hline $\mathrm{C}$ & 9.716219000 & 13.598615000 & 3.81750900 \\
\hline $\mathrm{C}$ & 7.411892000 & 13.176753000 & 3.58270000 \\
\hline $\mathrm{C}$ & 10.499560000 & 9.944257000 & 4.14583900 \\
\hline $\mathrm{C}$ & 7.402873000 & 10.887965000 & 2.61950900 \\
\hline 0 & 10.385622000 & 8.092249000 & 6.36952200 \\
\hline 0 & 5.011249000 & 9.793717000 & 3.82180600 \\
\hline $\mathrm{N}$ & 8.439210000 & 7.271774000 & 5.40374600 \\
\hline $\mathrm{N}$ & 9.438936000 & 9.018963000 & 4.45474600 \\
\hline $\mathrm{N}$ & 6.306957000 & 7.947916000 & 4.39769700 \\
\hline
\end{tabular}




\begin{tabular}{|c|c|c|c|}
\hline $\mathrm{N}$ & 7.310664000 & 9.708830000 & 3.455877000 \\
\hline C & 9.505355000 & 8.132454000 & 5.50300200 \\
\hline C & 7.662628000 & 7.486498000 & 4.19419900 \\
\hline C & 8.361652000 & 8.713412000 & 3.5316260 \\
\hline C & 6.092160000 & 9.216474000 & 3.89117300 \\
\hline C & 8.327878000 & 6.092876000 & 6.23211000 \\
\hline $\mathrm{C}$ & 5.225184000 & 7.075114000 & 4.7885340 \\
\hline 0 & 8.583380000 & 6.496263000 & 9.09201900 \\
\hline 0 & 3.162938000 & 8.033865000 & 6.56605100 \\
\hline $\mathrm{N}$ & 6.282550000 & 6.130027000 & 9.2047450 \\
\hline $\mathrm{N}$ & 7.265382000 & 6.150852000 & 7.2051220 \\
\hline $\mathrm{N}$ & 4.115322000 & 6.665848000 & 8.1901350 \\
\hline $\mathrm{N}$ & 5.133974000 & 6.844131000 & 6.2130680 \\
\hline $\mathrm{C}$ & 7.493239000 & 6.290881000 & 8.5569340 \\
\hline $\mathrm{C}$ & 5.215888000 & 5.735378000 & 8.2977720 \\
\hline $\mathrm{C}$ & 5.891662000 & 5.811834000 & 6.89604700 \\
\hline $\mathrm{C}$ & 4.045778000 & 7.271867000 & 6.9516070 \\
\hline $\mathrm{C}$ & 6.199871000 & 5.923469000 & 10.63004400 \\
\hline $\mathrm{C}$ & 3.078009000 & 6.799961000 & 9.18312800 \\
\hline 0 & 7.485030000 & 7.902388000 & 12.2465850 \\
\hline 0 & 2.081632000 & 9.455709000 & 9.7448570 \\
\hline $\mathrm{N}$ & 5.355053000 & 8.703842000 & 12.76623400 \\
\hline $\mathrm{N}$ & 5.555755000 & 7.002825000 & 11.34549200 \\
\hline $\mathrm{N}$ & 3.194842000 & 9.303027000 & 11.78349800 \\
\hline $\mathrm{N}$ & 3.428030000 & 7.641192000 & 10.30776300 \\
\hline $\mathrm{C}$ & 6.247588000 & 7.884456000 & 12.1254150 \\
\hline $\mathrm{C}$ & 3.973755000 & 8.320474000 & 12.5003950 \\
\hline $\mathrm{C}$ & 4.115454000 & 7.148122000 & 11.48148500 \\
\hline $\mathrm{C}$ & 2.826389000 & 8.867636000 & 10.5221060 \\
\hline $\mathrm{C}$ & 5.720717000 & 9.566611000 & 13.86553100 \\
\hline $\mathrm{C}$ & 2.563721000 & 10.436819000 & 12.4233310 \\
\hline 0 & 7.882460000 & 11.482269000 & 13.58995000 \\
\hline 0 & 2.514822000 & 12.938500000 & 10.9586020 \\
\hline $\mathrm{N}$ & 6.237576000 & 13.109218000 & 13.61757900 \\
\hline $\mathrm{N}$ & 5.604323000 & 10.977046000 & 13.56774900 \\
\hline $\mathrm{N}$ & 4.180024000 & 13.676404000 & 12.41300300 \\
\hline $\mathrm{N}$ & 3.429043000 & 11.578508000 & 12.61068100 \\
\hline $\mathrm{C}$ & 6.688297000 & 11.819468000 & 13.5815960 \\
\hline $\mathrm{C}$ & 4.792572000 & 13.201026000 & 13.64223600 \\
\hline $\mathrm{C}$ & 4.347229000 & 11.708769000 & 13.71391400 \\
\hline $\mathrm{C}$ & 3.293273000 & 12.749577000 & 11.88706000 \\
\hline $\mathrm{C}$ & 7.126408000 & 14.242910000 & 13.7048650 \\
\hline $\mathrm{C}$ & 4.081735000 & 15.085097000 & 12.0808140 \\
\hline $\mathrm{N}$ & 11.163820000 & 9.859230000 & 8.46852400 \\
\hline $\mathrm{H}$ & 10.034208000 & 7.343826000 & 10.1945490 \\
\hline $\mathrm{H}$ & 10.744590000 & 7.802683000 & 11.62961500 \\
\hline $\mathrm{H}$ & 11.648829000 & 7.736215000 & 10.26151000 \\
\hline $\mathrm{H}$ & 12.149830000 & 9.668059000 & 8.67047200 \\
\hline $\mathrm{H}$ & 11.159142000 & 10.718816000 & 7.89591200 \\
\hline $\mathrm{H}$ & 10.828862000 & 9.120290000 & 7.8215020 \\
\hline $\mathrm{H}$ & 9.942272000 & 12.267126000 & 9.04960600 \\
\hline $\mathrm{H}$ & 9.224225000 & 12.379744000 & 10.49260500 \\
\hline $\mathrm{H}$ & 6.896128000 & 12.177134000 & 10.82603600 \\
\hline $\mathrm{H}$ & 7.143216000 & 10.427371000 & 10.6097210 \\
\hline $\mathrm{H}$ & 8.704110000 & 10.704777000 & 7.20076900 \\
\hline $\mathrm{H}$ & 7.621010000 & 13.799077000 & 9.01082700 \\
\hline $\mathrm{H}$ & 8.358031000 & 13.168915000 & 7.5217460 \\
\hline $\mathrm{H}$ & 5.974556000 & 13.792785000 & 7.14249100 \\
\hline $\mathrm{H}$ & 5.758947000 & 9.140257000 & 8.9293650 \\
\hline $\mathrm{H}$ & 4.522020000 & 9.972534000 & 7.97521100 \\
\hline $\mathrm{H}$ & 4.161172000 & 12.488708000 & 8.2780140 \\
\hline $\mathrm{H}$ & 5.126517000 & 13.380194000 & 9.45764300 \\
\hline $\mathrm{H}$ & 4.765829000 & 11.034447000 & 10.2244570 \\
\hline $\mathrm{H}$ & 6.966810000 & 11.896210000 & 5.8399480 \\
\hline $\mathrm{H}$ & 5.244287000 & 11.623857000 & 6.12336300 \\
\hline $\mathrm{H}$ & 6.764043000 & 9.361486000 & 14.12851400 \\
\hline $\mathrm{H}$ & 5.077770000 & 9.330919000 & 14.72694400 \\
\hline $\mathrm{H}$ & 5.647540000 & 4.993590000 & 10.8299320 \\
\hline
\end{tabular}




\begin{tabular}{rr}
$\mathrm{H}$ & 7.221692000 \\
$\mathrm{H}$ & 8.169151000 \\
$\mathrm{H}$ & 9.271832000 \\
$\mathrm{H}$ & 10.805162000 \\
$\mathrm{H}$ & 11.340777000 \\
$\mathrm{H}$ & 11.955681000 \\
$\mathrm{H}$ & 11.514975000 \\
$\mathrm{H}$ & 9.767280000 \\
$\mathrm{H}$ & 10.443382000 \\
$\mathrm{H}$ & 8.104449000 \\
$\mathrm{H}$ & 6.736111000 \\
$\mathrm{H}$ & 5.343535000 \\
$\mathrm{H}$ & 4.289590000 \\
$\mathrm{H}$ & 2.211887000 \\
$\mathrm{H}$ & 2.811070000 \\
$\mathrm{H}$ & 2.174821000 \\
$\mathrm{H}$ & 1.736417000 \\
$\mathrm{H}$ & 3.207765000 \\
$\mathrm{H}$ & 3.939246000 \\
$\mathrm{H}$ & 5.608296000 \\
$\mathrm{H}$ & 6.991371000 \\
$\mathrm{H}$ & 8.727675000 \\
$\mathrm{H}$ & 7.014609000 \\
$\mathrm{H}$ & 6.380213000 \\
$\mathrm{H}$ & 7.931513000 \\
$\mathrm{H}$ & 8.221485000 \\
$\mathrm{H}$ & 9.915453000 \\
$\mathrm{H}$ & 9.202364000 \\
$\mathrm{H}$ & 7.575101000 \\
$\mathrm{H}$ & 6.211803000 \\
$\mathrm{H}$ & 4.464831000 \\
$\mathrm{H}$ & 3.862980000 \\
$\mathrm{H}$ & 3.478534000 \\
$\mathrm{H}$ & 3.673061000 \\
$\mathrm{H}$ & 4.848443000 \\
$\mathrm{H}$ & 5.852169000 \\
$\mathrm{H}$ & 7.671518000 \\
$\mathrm{H}$ & 8.768738000 \\
$\mathrm{H}$ & 9.968767000 \\
$\mathrm{H}$ & 10.253647000 \\
$\mathrm{H}$ & 6.577686000 \\
$\mathrm{O}$ & 9.202366000 \\
$\mathrm{H}$ & 8.756859000 \\
$\mathrm{H}$ & 8.507866000 \\
& \\
\hline
\end{tabular}

5.838012000

5.217105000

5.985862000

9.803437000

9.715672000

14.080415000

15.230916000

17.314173000

15.668571000

13.865272000

14.939871000

6.108972000

7.551149000

7.256024000

5.800667000

10.122783000

10.754759000

15.207742000

15.660419000

17.511373000

18.389835000

16.102113000

15.635079000

11.205551000

10.623854000

9.562126000

17.005211000

17.897956000

17.736550000

16.704808000

13.808363000

11.430961000

8.038896000

6.201085000

4.733170000

4.869941000

6.577881000

8.507768000

11.931928000

14.245200000

9.555679000

9.818595000

10.600045000

9.082410000
11.015103000

5.585624000

6.777712000

3.097988000

4.809466000

6.314179000

5.007822000

10.195729000

10.445732000

14.020386000

14.459099000

4.274364000

4.474264000

8.690883000

9.557823000

13.403276000

11.779279000

11.431022000

13.008638000

8.029128000

8.766410000

3.666964000

3. 943785000

2. 387222000

1.690864000

8.475764000

5.656027000

7.832005000

11.105747000

12.873312000

14.497840000

14.660905000

13.440719000

11.821669000

8.562727000

6.329253000

3.574496000

2.530700000

2.335961000

3.108892000

6.611253000

12.069249000

12.512186000

12.122023000

\section{Compound 2a}

$\begin{array}{ll}38 & \\ \text { Pt } & -0.857049000 \\ \mathrm{~N} & -2.278897000 \\ \mathrm{Cl} & -0.554603000 \\ \mathrm{~N} & -1.111366000 \\ \mathrm{~N} & 0.613241000 \\ \mathrm{C} & -2.116588000 \\ \mathrm{C} & -1.757122000 \\ \mathrm{C} & -3.520338000 \\ \mathrm{C} & -2.080062000 \\ \mathrm{H} & -3.537266000 \\ \mathrm{H} & -3.776327000 \\ \mathrm{H} & -1.789069000 \\ \mathrm{H} & -0.736875000 \\ \mathrm{H} & -1.064498000 \\ \mathrm{C} & -3.095617000 \\ \mathrm{H} & -2.318740000 \\ \mathrm{C} & -2.730596000 \\ \mathrm{C} & -4.503834000 \\ \mathrm{H} & -3.060277000 \\ \mathrm{C} & -2.768542000\end{array}$

1.827943000

0.711373000

0.067455000

3. 398695000

2.868323000

4. 484656000

5.195823000

3. 881145000

5. 490084000

3. 164674000

3. 343393000

4. 469257000

5.608923000

5.906780000

6.615476000

4. 971641000

7. 331445000

6.009484000

7. 327139000

6.325853000
0.692884000

$-0.292910000$

2.168576000

$-0.646890000$

1.690817000

$-0.358713000$

0.950255000

$-0.241366000$

$-1.521796000$

0.595186000

$-1.169315000$

1.777555000

0.884610000

$-1.616588000$

$-1.260814000$

$-2.464260000$

0.049497000

$-1.148341000$

$-2.098628000$

1.211432000 


$\begin{array}{lr}\mathrm{H} & -3.442182000 \\ \mathrm{H} & -1.727978000 \\ \mathrm{C} & -4.538982000 \\ \mathrm{H} & -4.776137000 \\ \mathrm{H} & -5.242307000 \\ \mathrm{C} & -4.178259000 \\ \mathrm{H} & -2.499739000 \\ \mathrm{H} & -4.909909000 \\ \mathrm{H} & -4.218940000 \\ \mathrm{H} & -0.200338000 \\ \mathrm{H} & -1.340658000 \\ \mathrm{H} & -5.541846000 \\ \mathrm{H} & -1.988789000 \\ \mathrm{H} & -2.422061000 \\ \mathrm{H} & 0.312132000 \\ \mathrm{H} & 1.448980000 \\ \mathrm{H} & -3.188543000 \\ \mathrm{H} & 0.900598000\end{array}$
8.149180000
7.780074000
5.005801000
5.507628000
6.807194000
5.722660000
6.829806000
6.519112000
5.014219000
3.852138000
3.002782000
4.561902000
0.473866000
$-0.172138000$
3.792993000
2.996922000
1.176241000
2.340459000
0.240612000
$-0.031114000$
0.015414000
$-2.090154000$
$-0.975508000$
1. 327116000
2.151168000
1.531363000
2.169408000
$-0.788025000$
$-1.566785000$
0.096283000
$-1.245424000$
0.205658000
2.009299000
1.113735000
$-0.353568000$
2. 520574000

\section{Compound 2a@CB7}

\section{4}

Pt $\quad 10.734967000$

$\mathrm{Cl} \quad 12.000048000$

$\mathrm{N} \quad 10.174525000$

$\mathrm{N} \quad 9.645798000$

C $\quad 8.220507000$

C $\quad 7.364452000$

C $\quad 8.152302000$

C $\quad 7.685569000$

C $\quad 5.904426000$

C $\quad 6.695440000$

C $\quad 6.226942000$

C $\quad 5.834675000$

C $\quad 6.169064000$

C $\quad 5.375190000$

o $\quad 9.490228000$

O $\quad 4.168369000$

$\mathrm{N} \quad 8.521320000$

$\mathrm{N} \quad 7.384958000$

$\mathrm{N} \quad 6.388689000$

$\mathrm{N} \quad 5.257388000$

C $\quad 8.555588000$

C $\quad 7.296951000$

C $\quad 6.536918000$

C $\quad 5.164636000$

9.668524000

6.578007000

11.216835000

5.857001000

10.290431000

9.623998000

8.138724000

7.475045000

10.455506000

9.376061000

8.912025000

7.029942000

11.133225000

8.034174000

11.310315000

6.196555000

10.063269000

10.482655000

8.065626000

8.349315000

10.677997000
10.357941000

8.740792000

11.112200000

11.831490000

11.563035000

11.296643000

10.344418000

12.802582000

11.062721000

10.105385000

12.563235000

9.844674000

11.343449000

12.304404000

13.937446000

15.939083000

15.861760000

14.694250000

16.539171000

15.558221000

14.747151000

16.619138000

15.854767000

15.998361000

16.333574000

17.293067000

14.352116000

15.959301000

15.213475000

16.048391000

15.826564000

16.675444000

15.120786000

16.271089000

16.860254000

16.127441000

14.498502000

15.420794000

11.693977000

13.337248000

11.291138000

13.398874000

11.975509000

14.029366000

12.084857000
10.234190000

11.325584000

12.067384000

9.262700000

8.856760000

10.099791000

7.929771000

8.117503000

9.678903000

7.500081000

7.692474000

8.745559000

6.759188000

8.945554000

11.623176000

9.418763000

10.766633000

12.289858000

9.786624000

11.440695000

11.566998000

10.915150000

12.040409000

10.133927000

10.023206000

8.567362000

8.611478000

6.105605000

6.659083000

8.611343000

5.663570000

7.618434000

8.023478000

6.273170000

7.642710000

6.430679000

5.733515000

4.281410000

6.392316000

3.483645000

4.459895000

5.057118000

3.218680000

4.043944000

5.403294000 
9.496970000

9.705125000

7.403335000

10.388544000

7.410553000

10.183577000

4.947632000

8.273412000

9.280794000

6.180603000

7.247869000

9.329354000

7.518954000

8.280110000

6.009986000

8.070192000

5.053977000

8.294105000

3.022753000

5.986590000

6.991802000

3.867734000

4.904215000

7.208436000

4.913469000

5.610706000

3.847626000

5.876851000

2.800225000

7.167233000

1.941351000

5.029284000

5.254375000

2.982487000

3.133881000

5.951371000

3.663498000

3.816203000

2.621162000

5.384446000

2.427116000

7.714302000

2.456727000

6.249573000

5.406553000

4.125875000

3.377644000

6.574976000

4.828996000

4.234274000

3.240427000

7.242511000

4.115801000

11.394956000

9.261018000

10.102823000

10.871797000

12.315189000

11.487556000

10.784652000

10.175900000

9.656056000

7.426617000

7.751443000

8.784763000

7.748361000

8.308471000

5.860665000
12.080384000

13.549175000

13.133265000

9.888189000

10.875268000

7.927568000

9.854510000

7.167588000

8.995305000

7.993976000

9.690860000

8.024549000

7.474287000

8.694648000

9.249801000

5.985172000

7.146601000

6.378083000

8.196915000

6.070045000

6.088741000

6.744429000

6.873342000

6.206604000

5.748917000

5.805783000

7.368479000

5.895139000

6.887220000

7.792761000

9.589521000

8.562827000

6.999112000

9.322856000

7.673784000

7.791153000

8.269493000

7.138824000

8.936437000

9.372307000

10.492569000

11.038249000

13.050020000

12.838415000

10.794220000

13.667099000

11.563748000

11.505732000

13.080505000

11.642439000

12.786580000

13.879839000

15.084259000

9.610423000

10.780892000

12.139041000

10.845878000

9.182443000

10.331369000

8.888595000

12.109244000

12.672709000

12.153384000

10.410385000

10.510767000

13.686421000

13.006082000

13.456686000
3.370434000

3.846554000

3.579872000

4.301549000

2. 551024000

6.406138000

3. 678366000

5.326309000

4.542278000

4.344952000

3. 361554000

5.523394000

4.129654000

3.527864000

3.790828000

6.132119000

4.667824000

8.992467000

6.434974000

9.075010000

7.084227000

8.046048000

6.076268000

8.444207000

8.152866000

6.758390000

6.815410000

10.503970000

9.007591000

12.262706000

9.599300000

12.770313000

11.196145000

11.664561000

10.171474000

12.088446000

12.394059000

11.330306000

10.388357000

13.909635000

12.300893000

13.572260000

10.964707000

13.366666000

13.651329000

12.465070000

12.504262000

13.525362000

13.581129000

13.674129000

11.872240000

13.479217000

12.187909000

8.431082000

12.410130000

12.065827000

12.767751000

8.563964000

7.699423000

8.022499000

8.423199000

9.861491000

10.787803000

10.625262000

7.044339000

8.773050000

7.230909000

7.166318000 


\begin{tabular}{|c|c|}
\hline $\mathrm{H}$ & 6.186925000 \\
\hline $\mathrm{H}$ & 4.791626000 \\
\hline $\mathrm{H}$ & 4.322259000 \\
\hline $\mathrm{H}$ & 5.405816000 \\
\hline $\mathrm{H}$ & 5.300898000 \\
\hline $\mathrm{H}$ & 6.771871000 \\
\hline $\mathrm{H}$ & 5.136047000 \\
\hline $\mathrm{H}$ & 6.394959000 \\
\hline $\mathrm{H}$ & 4.673793000 \\
\hline $\mathrm{H}$ & 5.303183000 \\
\hline $\mathrm{H}$ & 6.893733000 \\
\hline $\mathrm{H}$ & 7.876716000 \\
\hline $\mathrm{H}$ & 8.993696000 \\
\hline $\mathrm{H}$ & 10.768362000 \\
\hline $\mathrm{H}$ & 11.173136000 \\
\hline $\mathrm{H}$ & 11.971797000 \\
\hline $\mathrm{H}$ & 11.509150000 \\
\hline $\mathrm{H}$ & 9.751850000 \\
\hline $\mathrm{H}$ & 10.556122000 \\
\hline $\mathrm{H}$ & 8.205178000 \\
\hline $\mathrm{H}$ & 6.985777000 \\
\hline $\mathrm{H}$ & 5.155493000 \\
\hline $\mathrm{H}$ & 4.148964000 \\
\hline $\mathrm{H}$ & 1.969332000 \\
\hline $\mathrm{H}$ & 2.484676000 \\
\hline $\mathrm{H}$ & 1.999174000 \\
\hline $\mathrm{H}$ & 1.636448000 \\
\hline $\mathrm{H}$ & 3.223851000 \\
\hline $\mathrm{H}$ & 4.062878000 \\
\hline $\mathrm{H}$ & 5.597236000 \\
\hline $\mathrm{H}$ & 6.961464000 \\
\hline $\mathrm{H}$ & 8.702571000 \\
\hline $\mathrm{H}$ & 6.994841000 \\
\hline $\mathrm{H}$ & 6.409486000 \\
\hline $\mathrm{H}$ & 7.986619000 \\
\hline $\mathrm{H}$ & 8.537226000 \\
\hline $\mathrm{H}$ & 9.885380000 \\
\hline $\mathrm{H}$ & 9.160088000 \\
\hline $\mathrm{H}$ & 7.523832000 \\
\hline $\mathrm{H}$ & 6.407980000 \\
\hline $\mathrm{H}$ & 4.681395000 \\
\hline $\mathrm{H}$ & 3.651076000 \\
\hline $\mathrm{H}$ & 3.077901000 \\
\hline $\mathrm{H}$ & 3.366331000 \\
\hline $\mathrm{H}$ & 4.481886000 \\
\hline $\mathrm{H}$ & 5.537441000 \\
\hline $\mathrm{H}$ & 7.485939000 \\
\hline $\mathrm{H}$ & 8.769802000 \\
\hline $\mathrm{H}$ & 10.007131000 \\
\hline $\mathrm{H}$ & 10.240157000 \\
\hline $\mathrm{H}$ & 6.659832000 \\
\hline
\end{tabular}

8.944037000

9.660869000 12.151605000 13.179017000 10.874780000 11.526774000 11.167843000 9.082958000 9.172910000 4.977493000 5.793632000 5.132206000 5.814669000 9.731186000 9.640030000 14.088248000 15.197241000 17.419759000 15.835353000 13.394754000 14.538985000 6.193149000 7.667827000 7.390267000 5.885759000 10.203192000 10.878572000 15.295373000 15.631261000 17.381199000 18.293786000 16.050933000 15.574604000 11.217235000 10.613205000

9.461350000 16.997427000 17.922694000 17.667235000 16.432791000 13.683297000 11.458472000 7.975905000 6.180414000 4.766792000 4.870520000 6.597124000 8.483503000 11.838785000 14.184133000 9.229518000
9.269639000 8.447915000 8.665647000 9.613069000 10.578601000 5.856852000 6.424137000 14.218372000 14.724808000 10.707699000 10.899770000 5.465371000 6.696324000 3.279480000 5.024768000 6.306333000 4.972816000 10.171342000 10.427575000 13.674422000 14.323527000 4.126393000 4.335885000 8.500547000 9.336708000 13.273395000 11.647503000 11.587661000 13.141059000 8.086476000 8.817979000 3.675048000 3.969835000 2.265930000 1. 651048000 8.461904000 5.623527000 7.779245000 11.157487000 12.966785000 14.489570000 14.588488000 13.277861000 11.629595000 8.396973000 6.183648000 3.466550000 2.565486000 2.426932000 3.125945000 6.836324000

\section{Compound 2a_aq@CB7}

$\begin{array}{lr}\text { 166 } & \\ \text { Pt } & 10.720981000 \\ \text { N } & 10.166783000 \\ \text { N } & 9.674931000 \\ \text { C } & 8.239074000 \\ \text { C } & 7.393802000 \\ \text { C } & 8.153824000 \\ \text { C } & 7.712116000 \\ \text { C } & 5.927677000 \\ \text { C } & 6.690334000 \\ \text { C } & 6.247188000 \\ \text { C } & 5.838147000 \\ \text { C } & 6.170063000\end{array}$

10.375008000

11.144279000

11.818255000

11.551612000

11.292326000

10.334132000

12.798666000

11.072921000

10.110306000

12.572306000

9.856220000

11.354465000
10.210937000
12.037229000
9.230772000
8.844863000
10.096673000
7.918044000
8.112226000
9.687341000
7.501187000
7.700486000
8.753849000
6.766268000 


\begin{tabular}{|c|c|c|c|}
\hline $\mathrm{C}$ & 5.404326000 & 12.320746000 & 8.960734000 \\
\hline 0 & 9.481736000 & 13.940564000 & 11.596701000 \\
\hline O & 4.159318000 & 15.937916000 & 9.419911000 \\
\hline $\mathrm{N}$ & 8.514762000 & 15.876509000 & 10.767276000 \\
\hline $\mathrm{N}$ & 7.378107000 & 14.691926000 & 12.276555000 \\
\hline $\mathrm{N}$ & 6.379641000 & 16.540279000 & 9.785866000 \\
\hline $\mathrm{N}$ & 5.248186000 & 15.565347000 & 11.443526000 \\
\hline $\mathrm{C}$ & 8.547449000 & 14.752866000 & 11.553250000 \\
\hline $\mathrm{C}$ & 7.286160000 & 16.626711000 & 10.915685000 \\
\hline $\mathrm{C}$ & 6.530006000 & 15.856582000 & 12.039249000 \\
\hline C & 5.155166000 & 16.000754000 & 10.135041000 \\
\hline $\mathrm{C}$ & 9.661051000 & 16.354887000 & 10.026725000 \\
\hline C & 6.568386000 & 17.291213000 & 8.564546000 \\
\hline 0 & 11.194412000 & 14.352768000 & 8.628757000 \\
\hline 0 & 5.856492000 & 15.949707000 & 6.104617000 \\
\hline $\mathrm{N}$ & 10.284218000 & 15.216543000 & 6.669901000 \\
\hline $\mathrm{N}$ & 9.622063000 & 16.067510000 & 8.615530000 \\
\hline $\mathrm{N}$ & 8.139025000 & 15.829738000 & 5.663325000 \\
\hline $\mathrm{N}$ & 7.469716000 & 16.673092000 & 7.619330000 \\
\hline $\mathrm{C}$ & 10.443779000 & 15.128110000 & 8.034677000 \\
\hline $\mathrm{C}$ & 9.374019000 & 16.275588000 & 6.276214000 \\
\hline C & 8.905484000 & 16.869556000 & 7.642215000 \\
\hline $\mathrm{C}$ & 7.028169000 & 16.124660000 & 6.430135000 \\
\hline C & 11.126547000 & 14.493787000 & 5.750985000 \\
\hline $\mathrm{C}$ & 8.037371000 & 15.424622000 & 4.280468000 \\
\hline 0 & 11.260141000 & 11.693523000 & 6.434572000 \\
\hline 0 & 6.198285000 & 13.340503000 & 3.480026000 \\
\hline $\mathrm{N}$ & 10.053907000 & 11.289158000 & 4.476364000 \\
\hline $\mathrm{N}$ & 10.471004000 & 13.396239000 & 5.075302000 \\
\hline $\mathrm{N}$ & 8.067087000 & 11.976728000 & 3.222381000 \\
\hline $\mathrm{N}$ & 8.350627000 & 14.032407000 & 4.043271000 \\
\hline $\mathrm{C}$ & 10.651701000 & 12.083362000 & 5.427724000 \\
\hline $\mathrm{C}$ & 9.497417000 & 12.078995000 & 3.380972000 \\
\hline $\mathrm{C}$ & 9.706005000 & 13.547796000 & 3.856373000 \\
\hline $\mathrm{C}$ & 7.404885000 & 13.136751000 & 3.577605000 \\
\hline $\mathrm{C}$ & 10.380721000 & 9.885675000 & 4.320652000 \\
\hline $\mathrm{C}$ & 7.414063000 & 10.876805000 & 2.551972000 \\
\hline 0 & 10.163259000 & 7.921801000 & 6.421372000 \\
\hline 0 & 4.948108000 & 9.867421000 & 3.681861000 \\
\hline $\mathrm{N}$ & 8.260440000 & 7.163385000 & 5.327645000 \\
\hline $\mathrm{N}$ & 9.272017000 & 8.992653000 & 4.553716000 \\
\hline $\mathrm{N}$ & 6.172753000 & 7.999557000 & 4.344509000 \\
\hline $\mathrm{N}$ & 7.247260000 & 9.693489000 & 3.363289000 \\
\hline $\mathrm{C}$ & 9.314128000 & 8.020069000 & 5.532892000 \\
\hline $\mathrm{C}$ & 7.509531000 & 7.475571000 & 4.129869000 \\
\hline $\mathrm{C}$ & 8.276054000 & 8.694882000 & 3.533009000 \\
\hline $\mathrm{C}$ & 6.007487000 & 9.257000000 & 3.792215000 \\
\hline $\mathrm{C}$ & 8.051046000 & 5.980083000 & 6.130994000 \\
\hline $\mathrm{C}$ & 5.041993000 & 7.155021000 & 4.662015000 \\
\hline 0 & 8.270187000 & 6.366119000 & 8.992442000 \\
\hline 0 & 3.016999000 & 8.215821000 & 6.430236000 \\
\hline $\mathrm{N}$ & 5.962101000 & 6.060539000 & 9.069054000 \\
\hline $\mathrm{N}$ & 6.970856000 & 6.086911000 & 7.080483000 \\
\hline $\mathrm{N}$ & 3.850368000 & 6.753423000 & 8.038552000 \\
\hline $\mathrm{N}$ & 4.888304000 & 6.878515000 & 6.069225000 \\
\hline $\mathrm{C}$ & 7.185124000 & 6.198031000 & 8.441412000 \\
\hline $\mathrm{C}$ & 4.888850000 & 5.749963000 & 8.143483000 \\
\hline $\mathrm{C}$ & 5.589591000 & 5.807545000 & 6.750773000 \\
\hline $\mathrm{C}$ & 3.835225000 & 7.380224000 & 6.809216000 \\
\hline $\mathrm{C}$ & 5.848483000 & 5.883713000 & 10.497383000 \\
\hline $\mathrm{C}$ & 2.781466000 & 6.898626000 & 8.998308000 \\
\hline 0 & 7.151832000 & 7.783240000 & 12.245013000 \\
\hline 0 & 1.930655000 & 9.601451000 & 9.596507000 \\
\hline $\mathrm{N}$ & 5.018147000 & 8.562560000 & 12.757393000 \\
\hline $\mathrm{N}$ & 5.232140000 & 6.990430000 & 11.190361000 \\
\hline $\mathrm{N}$ & 2.966462000 & 9.324742000 & 11.663064000 \\
\hline $\mathrm{N}$ & 3.117765000 & 7.681005000 & 10.164267000 \\
\hline $\mathrm{C}$ & 5.935016000 & 7.784221000 & 12.076089000 \\
\hline
\end{tabular}




\begin{tabular}{|c|c|c|c|}
\hline $\mathrm{C}$ & 3.649518000 & 8.270430000 & 12.388336000 \\
\hline C & 3.795066000 & 7.139687000 & 11.32348400 \\
\hline C & 2.606777000 & 8.943743000 & 10.38486100 \\
\hline C & 5.379214000 & 9.368186000 & 13.89763300 \\
\hline C & 2.417974000 & 10.496519000 & 12.30087300 \\
\hline O & 7.710949000 & 11.029157000 & 13.54634500 \\
\hline 0 & 2.450466000 & 13.053800000 & 10.9650220 \\
\hline $\mathrm{N}$ & 6.248396000 & 12.833075000 & 13.3529420 \\
\hline $\mathrm{N}$ & 5.403348000 & 10.790818000 & 13.6427190 \\
\hline $\mathrm{N}$ & 4.122618000 & 13.667779000 & 12.4632830 \\
\hline $\mathrm{N}$ & 3.373485000 & 11.564345000 & 12.5004160 \\
\hline $\mathrm{C}$ & 6.571852000 & 11.499782000 & 13.5084510 \\
\hline C & 4.829033000 & 13.077922000 & 13.5752300 \\
\hline $\mathrm{C}$ & 4.232238000 & 11.641003000 & 13.66890500 \\
\hline $\mathrm{C}$ & 3.234902000 & 12.788778000 & 11.8712690 \\
\hline $\mathrm{C}$ & 7.242287000 & 13.873246000 & 13.46412000 \\
\hline $\mathrm{C}$ & 4.109426000 & 15.086215000 & 12.1916330 \\
\hline $\mathrm{N}$ & 11.390557000 & 9.597886000 & 8.4220270 \\
\hline $\mathrm{H}$ & 9.264579000 & 10.800410000 & 12.40042900 \\
\hline $\mathrm{H}$ & 10.073641000 & 12.170999000 & 12.0251170 \\
\hline $\mathrm{H}$ & 10.880154000 & 10.907202000 & 12.73203400 \\
\hline $\mathrm{H}$ & 12.328022000 & 9.201434000 & 8.52968000 \\
\hline $\mathrm{H}$ & 11.456518000 & 10.314172000 & 7.67991700 \\
\hline $\mathrm{H}$ & 10.794784000 & 8.858257000 & 8.02042900 \\
\hline $\mathrm{H}$ & 10.201724000 & 12.068874000 & 8.3778530 \\
\hline $\mathrm{H}$ & 9.701750000 & 12.673264000 & 9.81154600 \\
\hline $\mathrm{H}$ & 7.470123000 & 12.146657000 & 10.78569900 \\
\hline $\mathrm{H}$ & 7.773191000 & 10.399706000 & 10.6168070 \\
\hline $\mathrm{H}$ & 8.779300000 & 10.494518000 & 7.02714700 \\
\hline $\mathrm{H}$ & 7.787551000 & 13.680386000 & 8.76906100 \\
\hline $\mathrm{H}$ & 8.328142000 & 12.998320000 & 7.22039400 \\
\hline $\mathrm{H}$ & 5.885735000 & 13.470010000 & 7.1786630 \\
\hline $\mathrm{H}$ & 6.186219000 & 8.951557000 & 9.27387400 \\
\hline $\mathrm{H}$ & 4.791045000 & 9.682664000 & 8.4649310 \\
\hline $\mathrm{H}$ & 4.347701000 & 12.179361000 & 8.68985400 \\
\hline $\mathrm{H}$ & 5.449814000 & 13.193859000 & 9.6292910 \\
\hline $\mathrm{H}$ & 5.331388000 & 10.889761000 & 10.59274200 \\
\hline $\mathrm{H}$ & 6.766509000 & 11.533269000 & 5.85873400 \\
\hline $\mathrm{H}$ & 5.132438000 & 11.189503000 & 6.4407190 \\
\hline $\mathrm{H}$ & 6.390023000 & 9.075733000 & 14.20228000 \\
\hline $\mathrm{H}$ & 4.670948000 & 9.168950000 & 14.71485200 \\
\hline $\mathrm{H}$ & 5.268260000 & 4.969667000 & 10.6982860 \\
\hline $\mathrm{H}$ & 6.863880000 & 5.774491000 & 10.89474300 \\
\hline $\mathrm{H}$ & 7.856033000 & 5.129566000 & 5.46174200 \\
\hline $\mathrm{H}$ & 8.972612000 & 5.804996000 & 6.69688100 \\
\hline $\mathrm{H}$ & 10.766574000 & 9.730596000 & 3.30074400 \\
\hline $\mathrm{H}$ & 11.161954000 & 9.636399000 & 5.04688200 \\
\hline $\mathrm{H}$ & 11.960636000 & 14.081038000 & 6.32835400 \\
\hline $\mathrm{H}$ & 11.509481000 & 15.186566000 & 4.9885220 \\
\hline $\mathrm{H}$ & 9.736035000 & 17.441570000 & 10.17357900 \\
\hline $\mathrm{H}$ & 10.551068000 & 15.863905000 & 10.43437900 \\
\hline $\mathrm{H}$ & 8.205847000 & 13.387920000 & 13.65366100 \\
\hline $\mathrm{H}$ & 6.990151000 & 14.529749000 & 14.31183000 \\
\hline $\mathrm{H}$ & 5.141192000 & 6.202866000 & 4.11794800 \\
\hline $\mathrm{H}$ & 4.139735000 & 7.680620000 & 4.32945700 \\
\hline $\mathrm{H}$ & 1.953835000 & 7.407068000 & 8.49131900 \\
\hline $\mathrm{H}$ & 2.460391000 & 5.897876000 & 9.32428000 \\
\hline $\mathrm{H}$ & 1.992715000 & 10.209056000 & 13.2749980 \\
\hline $\mathrm{H}$ & 1.626307000 & 10.885430000 & 11.65042900 \\
\hline $\mathrm{H}$ & 3.215396000 & 15.298252000 & 11.5949040 \\
\hline $\mathrm{H}$ & 4.058337000 & 15.629204000 & 13.14705000 \\
\hline $\mathrm{H}$ & 5.588182000 & 17.373914000 & 8.0815670 \\
\hline $\mathrm{H}$ & 6.947674000 & 18.294279000 & 8.81208400 \\
\hline $\mathrm{H}$ & 8.708230000 & 16.054095000 & 3.6763580 \\
\hline $\mathrm{H}$ & 6.999186000 & 15.579858000 & 3.9658970 \\
\hline $\mathrm{H}$ & 6.414674000 & 11.219719000 & 2.2621860 \\
\hline $\mathrm{H}$ & 7.993677000 & 10.613418000 & 1.65474400 \\
\hline $\mathrm{H}$ & 8.531806000 & 9.445064000 & 8.44520000 \\
\hline
\end{tabular}




$\begin{array}{rrrr}\mathrm{H} & 9.887550000 & 16.998642000 & 5.626476000 \\ \mathrm{H} & 9.145986000 & 17.934279000 & 7.773021000 \\ \mathrm{H} & 7.506647000 & 17.675849000 & 11.158757000 \\ \mathrm{H} & 6.406761000 & 16.428242000 & 12.970188000 \\ \mathrm{H} & 4.687666000 & 13.679517000 & 14.485321000 \\ \mathrm{H} & 3.650886000 & 11.457315000 & 14.584396000 \\ \mathrm{H} & 3.068745000 & 7.977299000 & 13.275361000 \\ \mathrm{H} & 3.338385000 & 6.184040000 & 11.621088000 \\ \mathrm{H} & 4.449911000 & 4.770038000 & 8.382983000 \\ \mathrm{H} & 5.515638000 & 4.873584000 & 6.174138000 \\ \mathrm{H} & 7.475301000 & 6.600264000 & 3.464666000 \\ \mathrm{H} & 8.770723000 & 8.483737000 & 2.573336000 \\ \mathrm{H} & 10.013247000 & 11.834116000 & 2.441598000 \\ \mathrm{H} & 10.250305000 & 14.179953000 & 3.140522000 \\ \mathrm{H} & 6.642032000 & 9.235712000 & 6.836695000 \\ \mathrm{O} & 11.806981000 & 8.936716000 & 11.300113000 \\ \mathrm{H} & 12.769230000 & 8.980504000 & 11.135608000 \\ \mathrm{H} & 11.536092000 & 8.021592000 & 11.091720000\end{array}$

